\title{
Interplay between collective effects and non-standard interactions of supernova neutrinos
}

\author{
A. Esteban-Pretel, ${ }^{1}$ R. Tomàs, ${ }^{2}$ and J. W. F. Valle ${ }^{1}$ \\ ${ }^{1}$ AHEP Group, Institut de Física Corpuscular - C.S.I.C/Universitat de València \\ Edifici Instituts d'Investigació, Apt. 22085, E-46071 València, Spain \\ ${ }^{2}$ II. Institut für theoretische Physik, Universität Hamburg, \\ Luruper Chaussee 149, 22761 Hamburg, Germany
}

(Dated: January 16, 2021)

\begin{abstract}
We consider the effect of non-standard neutrino interactions (NSI, for short) on the propagation of neutrinos through the supernova (SN) envelope within a three-neutrino framework and taking into account the presence of a neutrino background. We find that for given NSI parameters, with strength generically denoted by $\varepsilon_{i j}$, neutrino evolution exhibits a significant time dependence. For $\left|\varepsilon_{\tau \tau}\right| \gtrsim$ $10^{-3}$ the neutrino survival probability may become sensitive to the $\vartheta_{23}$ octant and the sign of $\varepsilon_{\tau \tau}$. In particular, if $\varepsilon_{\tau \tau} \gtrsim 10^{-2}$ an internal $I$-resonance may arise independently of the matter density. For typical values found in SN simulations this takes place in the same dense-neutrino region above the neutrinosphere where collective effects occur, in particular during the synchronization regime. This resonance may lead to an exchange of the neutrino fluxes entering the bipolar regime. The main consequences are (i) bipolar conversion taking place for normal neutrino mass hierarchy and (ii) a transformation of the flux of low-energy $\nu_{e}$, instead of the usual spectral swap.

PACS numbers: 13.15.+g, 14.60.Lm, 14.60.Pq, 14.60.St, 97.60.Bw
\end{abstract}

\section{INTRODUCTION}

Current neutrino data imply that neutrino have mass. Indeed reactor data by the KamLAND collaboration 1] and data from accelerator neutrino experiments $\mathrm{K} 2 \mathrm{~K}[2]$ and MINOS [3] not only confirm the neutrino flavor conversion discovered in the study of solar [4 7] and atmospheric neutrinos [8, 9] but also indicate that the underlying neutrino flavor conversion mechanism is oscillatory in both cases [10]. An updated review of the current status of neutrino oscillations is given in Refs. [11].

Theories of neutrino mass 12, 13 typically require that neutrinos have non-standard four-Fermi interactions ${ }^{1}$ which, for short, we denote as NSI 15 17]. These are natural outcome of neutrino mass models and can be of two types: flavor-changing (FC) and non-universal (NU). For example, generic seesaw-type models lead to a nontrivial structure of the lepton mixing matrix characterizing the charged and neutral current weak interactions

\footnotetext{
${ }^{1}$ Other non-standard neutrino properties such as electromagnetic transition moments may also be present [14].
}

implying an effective non-unitary form for the mixing matrix describing neutrino oscillations [12]. However, the expected magnitude of the unitarity violation and of the four-Fermi NSI effects is rather model-dependent. In the simplest high-scale seesaw models these are all negligible. In contrast, the 4-Fermi NSI effects induced by the charged and neutral current gauge interactions may be sizeable in low-scale seesaw schemes [18 25].

Alternatively, non-standard neutrino interactions may arise in models where neutrino masses are radiatively induced by low-scale loop effects [26 28], or directly "calculable" by renormalization group evolution [29] in some supergravity models.

It is important to realize that the strengths of nonstandard interactions need not be suppressed by the smallness of neutrino masses. Indeed, they may be very relevant for the propagation of supernova (SN) neutrinos and give rise to a novel type of resonant neutrino flavor conversion mechanism that can take place even in the limit of massless neutrinos $[17,30]^{2}$. Therefore we

\footnotetext{
${ }^{2}$ Similarly flavor and CP-violating effects can survive in the limit
} 
argue that, in addition to the precision determination of the oscillation parameters, it is necessary to test for sub-leading non-oscillation effects that could arise from non-standard neutrino interactions in upcoming neutrino experiments 31, 32]. Especially because NSI effects can in some cases fake genuine mixing effects [33, 34].

Here we concentrate on the impact of non-standard neutrino interactions on SN physics. The main motivation of the work is to reexamine the effect of non-standard neutrino interactions on supernova when the neutrino self-interaction is taken into account. The extreme conditions under which neutrinos propagate, since they are created in the SN core until they reach the Earth, may lead to strong matter effects. It is known that, in particular, the effect of small values of the NSI parameters can be dramatically enhanced in the inner strongly deleptonized regions [17, 30, 35, 36]. On the other hand, it has been recently pointed out 37 61] that in this region the large neutrino background could itself affect neutrino propagation significantly. In this paper we analyze the interplay between these two in principle coexisting effects, the NSI and the neutrino self-interaction, and the resulting consequences for neutrino evolution through the SN envelope.

The paper is organized as follows. In Sec. I we describe the equations of motion that govern neutrino propagation taking into account both NSI and self interaction effects. In Sec. III] we study the interplay between these two effects and analyze the conditions for different regimes of evolution to take place. In Sec. IV and V we describe in detail the salient features characterizing neutrino evolution in the different regions defined by the conditions given in the previous section. Finally, before summarizing in Sec. VII, we give, in Sec. VI, a time dependent discussion of the studied effects.

\section{EQUATIONS OF MOTION}

The equations of motion (EOMs) of the neutrinos traveling through the SN envelope can be written as

$$
\mathrm{i} \partial_{t} \varrho_{\mathbf{p}}=\left[\mathrm{H}_{\mathbf{p}}, \varrho_{\mathbf{p}}\right],
$$

of massless neutrinos [19, 20]. where $\rho_{\mathbf{p}}$ and $\bar{\rho}_{\mathbf{p}}$ represent the density matrices describing each (anti)neutrino mode. The diagonal entries are the usual occupation numbers whereas the off-diagonal terms encode phase information. The Hamiltonian for neutrinos has the form

$$
\mathrm{H}_{\mathbf{p}}=\Omega_{\mathbf{p}}+\mathrm{V}+\sqrt{2} G_{\mathrm{F}} \int \frac{\mathrm{d}^{3} \mathbf{q}}{(2 \pi)^{3}}\left(\varrho_{\mathbf{q}}-\bar{\varrho}_{\mathbf{q}}\right)\left(1-\mathbf{v}_{\mathbf{q}} \cdot \mathbf{v}_{\mathbf{p}}\right) .
$$

For antineutrinos the only difference is $\Omega_{\mathbf{p}} \rightarrow-\Omega_{\mathbf{p}}$.

The first term stands for the matrix of vacuum oscillation frequencies, $\Omega_{\mathbf{p}}=U \operatorname{diag}\left(m_{1}^{2}, m_{2}^{2}, m_{3}^{2}\right) / 2|\mathbf{p}| U^{\dagger}$ in the weak basis, where $U$ is the three-neutrino lepton mixing matrix 12 in the unitary approximation and PDG convention [62], with no $C P$ phases. We use $\Delta m_{21}^{2} \equiv m_{2}^{2}-m_{1}^{2}=7.65 \times 10^{-5} \mathrm{eV}^{2},\left|\Delta m_{31}^{2}\right| \equiv$ $\left|m_{3}^{2}-m_{1}^{2}\right|=2.40 \times 10^{-3} \mathrm{eV}^{2}, \sin ^{2} \vartheta_{12}=0.3$, as obtained in e.g. Ref [11]. We consider also $\sin ^{2} \vartheta_{13}=10^{-2}$ and three different values for $\vartheta_{23}$ in the allowed range at $3 \sigma, \sin ^{2} \vartheta_{23}=0.4,0.5$ and 0.6 , because our results depend sensitively on $\vartheta_{23}$. Given the values of $\Delta m^{2}$ one can define the two associated vacuum oscillation frequencies: $\omega_{\mathrm{H}} \equiv \Delta m_{31}^{2} / 2 E$ and $\omega_{\mathrm{L}} \equiv \Delta m_{21}^{2} / 2 E$, which, in the case of neutrinos with $E=20 \mathrm{MeV}$, take the values $\omega_{\mathrm{H}}=0.3 \mathrm{~km}^{-1}$ and $\omega_{\mathrm{L}}=0.01 \mathrm{~km}^{-1}$. In the top panel of Fig. 1 we represent $\omega_{\mathrm{H}}$ and $\omega_{L}$ for energies typical in $\mathrm{SNe}$, between $5 \mathrm{MeV}$ and $50 \mathrm{MeV}$, as yellow and light blue horizontal bands, respectively.

The second term of the Hamiltonian accounts for the interaction of neutrinos with matter and can be split into two pieces,

$$
\mathrm{V}=\mathrm{V}_{\mathrm{std}}+\mathrm{V}_{\mathrm{nsi}}
$$

The first term, $\mathrm{V}_{\text {std }}$, describes the standard interaction with matter and can be represented in the weak basis by $\mathrm{V}_{\mathrm{std}}=\sqrt{2} G_{\mathrm{F}} n_{B} \operatorname{diag}\left(Y_{e}, 0, Y_{\tau}^{\text {eff }}\right)$, with $G_{\mathrm{F}}$ the Fermi constant, $n_{B}$ the baryon density, and $Y_{e}=n_{e} / n_{B}$ stands for the electron fraction. We consider the following standard weak potential

$$
\mathrm{V}_{\text {std }}=\lambda(r) \operatorname{diag}\left(Y_{e}, 0, Y_{\tau}^{\mathrm{eff}}\right),
$$

with

$$
\lambda(r)=\lambda_{0}\left(\frac{R}{r}\right)^{3} .
$$

In the following we assume $R=10 \mathrm{~km}$. In the top panel of Fig. 1 we show two $\lambda(r)$ profiles for $\lambda_{1,0}=5 \times 10^{9} \mathrm{~km}^{-1}$ 


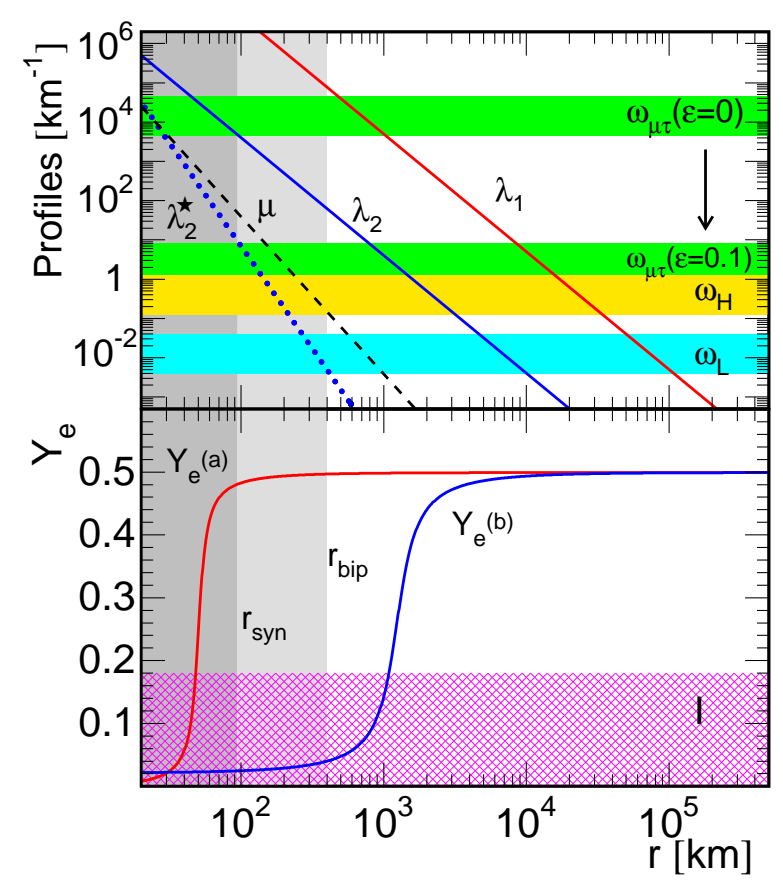

FIG. 1: Top panel: $\lambda(r)$, profiles as defined in Eq. (5), for $\lambda_{1,0}=5 \times 10^{9} \mathrm{~km}^{-1}\left(\lambda_{1}\right)$ and $\lambda_{2,0}=4 \times 10^{6} \mathrm{~km}^{-1}\left(\lambda_{2}\right)$ in solid red and blue lines, respectively; $\lambda_{2}^{\star}(r)$, as given by Eq. (21), is shown in blue dotted lines; $\mu(r)$, introduced in Eq. (13), for $\mu_{0}=7 \times 10^{5} \mathrm{~km}^{-1}$ in black dashed lines; the vacuum oscillation frequencies $\omega_{\mathrm{H}}$ (yellow band), $\omega_{L}$ (light blue band), and $\omega_{\mu \tau}^{\mathrm{nsi}}$, defined in Eq. (17), for $\varepsilon_{\tau \tau}=0$ and 0.1 (green bands), for energies between 5 and $50 \mathrm{MeV}$. The position of the synchronization and bipolar radii are also shown. Bottom: radial dependence of $Y_{e}$, given by Eq. (6), for two set of parameters: $a=0.24, b=0.165, r_{0}=50\left(1.2 \times 10^{3}\right) \mathrm{km}$, and $r_{s}=5\left(3 \times 10^{2}\right) \mathrm{km}$, for $Y_{e}^{\mathrm{a}}\left(Y_{e}^{\mathrm{b}}\right)$. The horizontal magenta band represents the $Y_{e}^{I}$ leading to an internal $I$-resonance for $\varepsilon_{\tau \tau} \leq 0.1$.

and $\lambda_{2,0}=4 \times 10^{6} \mathrm{~km}^{-1}$ denoted by $\lambda_{1}$ and $\lambda_{2}$, corresponding to typical early and late time profiles, respectively [53].

The first element in $\mathrm{V}^{\text {std }}$ represents the charged current potential and is proportional to the electron fraction, $Y_{e}$. According to the SN models, $Y_{e}$ is characterized by a transition from a few $\%$ in the inner most deleptonized layers until values around 0.5 in the outer envelope. Following Ref. [36] we parametrize it phenomenologically as

$$
Y_{e}(r)=a+b \arctan \left[\left(r-r_{0}\right) / r_{s}\right] \text {, }
$$

with $a=0.24$ and $b=0.165$. The parameters $r_{0}$ and $r_{s}$ describe where the rise takes place and how steep it is, respectively. In the bottom panel of Fig. 1 we show two $Y_{e}(r)$ profiles for two different choices of these parameters. The radius where $\lambda(r) Y_{e}(r)$ crosses the horizontal bands $\omega_{\mathrm{H}}\left(\omega_{\mathrm{L}}\right)$ determines the well known $H(L)$ Mikheyev-Smirnov-Wolfenstein (MSW) resonances 63, 64. For the $\lambda$ and $Y_{e}$ profiles shown in Fig. 1 and energies typical in SNe the position of both resonances $r_{\mathrm{H}}$ and $r_{\mathrm{L}}$ lie above $10^{3} \mathrm{~km}$.

The other non-zero element in $\mathrm{V}_{\text {std }}$ arises from radiative corrections to neutral-current $\nu_{\mu}$ and $\nu_{\tau}$ scattering. Although there are no $\mu$ nor $\tau$ leptons in normal matter, they appear as virtual states causing a shift $\Delta V_{\mu \tau}=\sqrt{2} G_{\mathrm{F}} Y_{\tau}^{\text {eff }} n_{B}$ between $\nu_{\mu}$ and $\nu_{\tau}$ due to the difference in their masses. It has the same effect on neutrino dispersion as real $\tau$ leptons with an effective abundance 65]

$$
Y_{\tau}^{\mathrm{eff}}=\frac{3 \sqrt{2} G_{\mathrm{F}} m_{\tau}^{2}}{(2 \pi)^{2}}\left[\ln \left(\frac{m_{W}^{2}}{m_{\tau}^{2}}\right)-1+\frac{Y_{n}}{3}\right]=2.7 \times 10^{-5}
$$

where $n_{e}=n_{p}$ and $Y_{n}=0.5$ was assumed. Analogously to the $H$ - and $L$-resonances, the radius where $\lambda(r) Y_{\tau}^{\mathrm{eff}} \approx \omega_{\mathrm{H}}$ or, equivalently $\lambda(r) \approx \omega_{\mu \tau} \equiv \omega_{\mathrm{H}} / Y_{\tau}^{\mathrm{eff}}$, defines the $\mu \tau$-resonance [66]. The upper green band in the top panel of Fig. 1 represents $\omega_{\mu \tau}$ for energies between 5 and $50 \mathrm{MeV}$. In the present analysis we have not considered the flavor non-universal radiative correction for neutrino refraction in the presence of a neutrino background 67.

In order to construct the term in the Hamiltonian describing the non-standard neutrino interactions with a fermion $f$ we parametrize them with the effective lowenergy four-fermion operator:

$$
\mathcal{L}_{N S I}=-\varepsilon_{\alpha \beta}^{f P} 2 \sqrt{2} G_{F}\left(\bar{\nu}_{\alpha} \gamma_{\mu} L \nu_{\beta}\right)\left(\bar{f} \gamma^{\mu} P f\right)
$$

where $P=L, R$ and $f$ is a first generation fermion: $e, u, d$. We neglect the possible NSI effect arising in $\nu-\nu$ interactions 68]. The coefficients $\varepsilon_{\alpha \beta}^{f P}$ denote the strength of the NSI between the neutrinos of flavors $\alpha$ and $\beta$ and the $P$-handed component of the fermion $f$. Throughout the article we assume neutrinos propagating across an unpolarized medium 69]. Therefore their evolution in matter will be affected by the vector coupling 
constant of the NSI, $\varepsilon_{\alpha \beta}^{f V}=\varepsilon_{\alpha \beta}^{f L}+\varepsilon_{\alpha \beta}^{f R} 3$, see Ref. [69]. We also consider $\varepsilon_{\alpha \beta}^{f} \in \Re$, neglecting possible $C P$ violation in the new interactions ${ }^{4}$. Under these assumptions $V_{n s i}$ can be expressed as,

$$
\left(\mathrm{V}_{\mathrm{nsi}}\right)_{\alpha \beta}=\sum_{f=e, u, d}\left(\mathrm{~V}_{\mathrm{nsi}}^{f}\right)_{\alpha \beta}=\sqrt{2} G_{F} N_{f} \varepsilon_{\alpha \beta}^{f},
$$

where $N_{f}$ represents the fermion $f$ number density. For definiteness and motivated by actual models, for example, those with broken $\mathrm{R}$ parity supersymmetry we take for $f$ the down-type quark. Therefore the NSI potential can be expressed as follows,

$$
\left(\mathrm{V}_{\mathrm{nsi}}\right)_{\alpha \beta}=\left(\mathrm{V}_{\mathrm{nsi}}^{d}\right)_{\alpha \beta}=\varepsilon_{\alpha \beta}^{d} \lambda(r)\left(2-Y_{e}\right) .
$$

From now on we will not explicitly write the superindex $d$. In order to further simplify the problem one can always redefine the diagonal NSI parameters so that $\varepsilon_{\mu \mu}=0$, since subtracting a matrix proportional to the identity leaves the physics involved in the neutrino oscillation unaffected. Thus, in principle at least five of the six independent $\varepsilon_{\alpha \beta}$ parameters should be taken into account. Nevertheless, the exhaustive description of NSI in SN neutrinos developed in Ref. [36] shows that the physics involved can be described in terms of $\varepsilon_{\tau \tau}^{\prime}$ and $\varepsilon_{e \tau}^{\prime}$, which are just a suitable combination of $\varepsilon$ 's. This motivates us to illustrate the interplay that could arise between collective effects and NSI by only considering two non-zero NSI parameters: $\varepsilon_{e \tau}$ and $\varepsilon_{\tau \tau}$, describing flavor-changing (FC) processes and non-universality (NU), respectively. Therefore the term in the Hamiltonian responsible for the interactions with matter can be written as

$$
\mathrm{V}=\lambda(r)\left(2-Y_{e}\right)\left(\begin{array}{ccc}
\frac{Y_{e}}{2-Y_{e}} & 0 & \varepsilon_{e \tau} \\
0 & 0 & 0 \\
\varepsilon_{e \tau} & 0 & \varepsilon_{\tau \tau}+\frac{Y_{\tau}^{\text {eff }}}{2-Y_{e}}
\end{array}\right) .
$$

The range of values for the NSI parameters we consider is for the off-diagonal term $10^{-5} \lesssim\left|\varepsilon_{e \tau}\right| \lesssim$ few $\times 10^{-3}$. This prevents any significant NSI-induced reduction of the electron fraction $Y_{e}$ during the core collapse [1], 72]. For the diagonal term we assume $\left|\varepsilon_{\tau \tau}\right| \lesssim 0.1$, allowed by the current experimental constraints 73 76].

\footnotetext{
${ }^{3}$ For the sake of simplicity we will omit the superindex $V$.

${ }^{4}$ Possible CP effects in the case of SUSY radiative corrections on $\mu-\tau$ neutrino refraction including R-parity breaking interactions have been considered in Ref. 70]
}

Finally, the third term in the Hamiltonian accounts for the collective flavor transformations induced by neutrinoneutrino interaction. In spherical symmetry the EOMs can be expressed as a closed set of differential equations along the radial direction 45, 49]. We solve them numerically as described in Ref. 45]. The factor $\left(1-\mathbf{v}_{\mathbf{q}} \cdot \mathbf{v}_{\mathbf{p}}\right)$ in the Hamiltonian, $\mathbf{v}_{\mathbf{p}}$ being the velocity, implies "multiangle effects" for neutrinos moving on different trajectories [38, 41, 77]. We consider, though, the single-angle approximation by launching all neutrinos with $45^{\circ}$ relative to the radial direction. This approximation is valid for the neutrino fluxes assumed in this analysis provided that the neutrino density exceeds the electron density. The strength of the neutrino-neutrino interaction can be parametrized by

$$
\mu_{0}=\sqrt{2} G_{\mathrm{F}}\left(F_{\bar{\nu}_{e}}^{R}-F_{\bar{\nu}_{x}}^{R}\right),
$$

where the fluxes are taken at the radius $R$. Following Ref. 45] we shall assume $\mu_{0}=7 \times 10^{5} \mathrm{~km}^{-1}$. In the single-angle approximation the radial dependence of the neutrino-neutrino interaction strength can be explicitly written as

$$
\mu(r)=\mu_{0} \frac{R^{4}}{r^{4}} \frac{1}{2-R^{2} / r^{2}} \approx \mu_{0} \frac{R^{4}}{2 r^{4}} .
$$

In the top panel of Fig. 1 we show the typical $\mu(r)$ profile for $\mu_{0}=7 \times 10^{5} \mathrm{~km}^{-1}$. One final property of SN neutrinos with important consequences for our study is the hierarchy of fluxes obtained in SN models. The typical conditions of the proto-neutron star lead to the following hierarchy of fluxes $F_{\nu_{e}}^{R}>F_{\bar{\nu}_{e}}^{R}>F_{\nu_{x}}^{R}$, with $\nu_{x}$ standing for $\nu_{\mu}, \nu_{\tau}, \bar{\nu}_{\mu}$ and $\bar{\nu}_{\tau}$. As in Ref. 45] we express the lepton asymmetry with the parameter $\epsilon=\left(F_{\nu_{e}}^{R}-F_{\bar{\nu}_{e}}^{R}\right) /\left(F_{\bar{\nu}_{e}}^{R}-F_{\bar{\nu}_{x}}^{R}\right)$. Throughout the analysis we shall consider $\epsilon=0.25$. The equal parts of the fluxes drop out of the EOMs, so as initial condition we use in the monoenergetic case $F_{\nu_{\mu}, \bar{\nu}_{\mu}, \nu_{\tau}, \bar{\nu}_{\tau}}^{R}=0$ and $F_{\nu_{e}}^{R}=(1+\epsilon) F_{\bar{\nu}_{e}}^{R}$.

\section{NON-STANDARD INTERACTIONS IN THE PRESENCE OF COLLECTIVE EFFECTS}

In the absence of NSI and collective effects the neutrino propagation through the SN envelope is basically determined at the well-known MSW resonances defined in Sec. III. They arise when the matter potential becomes 
of the same order as the kinetic terms in the Hamiltonian. The $L$-resonance occurs always for neutrinos whereas the $H$-one takes place for (anti)neutrinos for (inverted) normal mass hierarchy. For our matter profiles and the values of $\vartheta_{12}$ and $\vartheta_{13}$ both resonances are adiabatic [78, 79]. Moreover both involve electron neutrino flavor and happen in the outer layers of the SN envelope, see top panel of Fig. 1.

In addition, the $\mu \tau$-resonance is also adiabatic [66], but occurs between the $\nu_{\mu}$ and $\nu_{\tau}$ or $\bar{\nu}_{\mu}$ and $\bar{\nu}_{\tau}$ depending on the neutrino mass hierarchy and the $\vartheta_{23}$ octant. However, when considering the neutrino self-interaction this resonance can also cause significant modifications of the overall $\nu_{e}$ and $\bar{\nu}_{e}$ survival probabilities [53]. According to the discussion in the previous section the $\mu \tau$-resonance occurs at

$$
r_{\mu \tau} \approx R\left(\frac{\lambda_{0} Y_{\tau}^{\mathrm{eff}}}{\omega_{\mathrm{H}}}\right)^{1 / 3}=R\left(\frac{\lambda_{0}}{\omega_{\mu \tau}}\right)^{1 / 3} .
$$

Due to the smallness of $Y_{\tau}^{\text {eff }}$ the $\mu \tau$-resonance happens at deeper layers than the $H$ - and $L$-resonances. In particular, for $\omega_{\mathrm{H}}=0.3 \mathrm{~km}^{-1}, Y_{e}=0.5$, and $\lambda_{0}=5 \times 10^{9} \mathrm{~km}^{-1}$ $\left(4 \times 10^{6} \mathrm{~km}^{-1}\right) r_{\mu \tau}=770 \mathrm{~km}(71 \mathrm{~km})$, see the intersection between the upper green band and the profiles $\lambda_{1}(r)$ and $\lambda_{2}(r)$ in the top panel of Fig. 1

The consequence of the addition of an NSI term such as that of Eq. (10) is twofold. First, it will affect the MSW resonances. For the values assumed here the main effect on the $H$ - and $L$-resonances will be just a slight shift in the resonance point [36, 80]. The consequences for the $\mu \tau$-resonance can be more drastic. For sufficiently large values of $\left|\varepsilon_{\tau \tau}\right|$ a negative sign can change the resonance channel, from $\nu$ to $\bar{\nu}$ or viceversa, depending on the octant of $\vartheta_{23}$. On the other hand, it can significantly modify the position of the resonance. In the presence of NSI the $\mu \tau$-resonance happens at

$$
r_{\mu \tau} \approx R\left(\frac{\lambda_{0} Y_{\tau, \mathrm{nsi}}^{\mathrm{eff}}}{\omega_{\mathrm{H}}}\right)^{1 / 3}=R\left(\frac{\lambda_{0}}{\omega_{\mu \tau}^{\mathrm{nsi}}}\right)^{1 / 3},
$$

where we have defined

$$
\begin{aligned}
Y_{\tau, \mathrm{nsi}}^{\mathrm{eff}} & \equiv Y_{\tau}^{\mathrm{eff}}+\left(2-Y_{e}\right) \varepsilon_{\tau \tau}, \\
\omega_{\mu \tau}^{\mathrm{nsi}} & \equiv \omega_{\mathrm{H}} / Y_{\tau, \mathrm{nsi}}^{\mathrm{eff}} .
\end{aligned}
$$

In particular, for $\left|\varepsilon_{\tau \tau}\right|>Y_{\tau}^{\text {eff }} /\left(2-Y_{e}\right)$ the value of $\omega_{\mu \tau}^{\text {nsi }}$ decreases, and therefore $r_{\mu \tau}$ is pushed outwards with respect to the standard case. The lower green band in the top panel of Fig. 1 1 represents $\omega_{\mu \tau}^{\text {nsi }}$ for $\varepsilon_{\tau \tau}=0.1, Y_{e}=0.5$ and typical SN neutrino energies. For $E=20 \mathrm{MeV}$ and the matter profile corresponding to $\lambda_{2,0}$, the position of the $\mu \tau$-resonance moves out to a radius of $r_{\mu \tau} \approx 1.3 \times 10^{3} \mathrm{~km}$.

The second important consequence is that the new NSI terms can induce additional resonances [17]. If we consider the inner layers, defined as those where $r \ll r_{H}$, in the absence of neutrino self-interaction, one may have $H \approx \mathrm{V}$, as given in Eq. (11). One can see that a novel resonance, which we call $I$-resonance, $I$ standing for "internal", will arise when the condition $H_{e e}=H_{\tau \tau}$ is satisfied. This occurs when the value of the $\varepsilon_{\alpha \beta}$ is of the same order as the electron fraction $Y_{e}$ [17]. Current constraints on the $\varepsilon_{\alpha \beta}$ 's imply that small values of $Y_{e}$ are required for these NSI-induced internal resonances to occur. Hence this condition is only fulfilled in the most deleptonized inner layers, close to the neutrinosphere, where $Y_{e}$ reaches values of a few $\%[17,30,35,36$. If we neglect the contribution from $Y_{\tau}^{\text {eff }}$ the corresponding resonance condition can be written as

$$
Y_{e}^{I}=\frac{2 \varepsilon_{\tau \tau}}{1+\varepsilon_{\tau \tau}} .
$$

In the bottom panel of Fig. 1 we show as a horizontal band the range of $Y_{e}^{I}$ required for the $I$-resonance to take place for $\varepsilon_{\tau \tau} \leq 0.1$. One sees how for the $Y_{e}(r)$ profiles found in numerical simulations the resonance condition can only be satisfied in the inner layers. For typical values of $Y_{e}$ one expects to have the $I$-resonance for $\varepsilon_{\tau \tau} \gtrsim 10^{-2}$. Moreover, as seen in 36], the range of $\left|\varepsilon_{e \tau}\right|$ considered ensures adiabaticity. It must be noted that, in contrast to the standard $H$ - and $L$-resonances, related to the kinetic term, neither the density nor the energy enter explicitly into the resonance condition, which is determined only by the electron fraction $Y_{e}$. Moreover, in contrast to the standard resonances, the $I$-resonance occurs for both neutrinos and antineutrinos simultaneously [17.

At the same time, also in the internal region, the neutrino flux emerging from the supernova core is so dense that, neutrino-neutrino refraction can cause nonlinear flavor oscillation phenomena $[3760$. The crucial effect is a collective mode of pair transformations of the form $\nu_{e} \bar{\nu}_{e} \rightarrow \nu_{x} \bar{\nu}_{x}$, where $x$ represents some suitable superposition of $\nu_{\mu}$ and $\nu_{\tau}$. This pair-wise form of flavor transformation leaves the net flavor-lepton number flux un- 
changed. For the hierarchy of neutrino fluxes assumed this conversion occurs only when the neutrino mass hierarchy is inverted. Collective flavor transformations start after the synchronization phase, where $\mu\left(r_{\mathrm{syn}}\right) \approx$ $2 \omega_{\mathrm{H}} /(1-\sqrt{1+\epsilon})^{2}$, and extends a few hundred $\mathrm{km}$ in the so-called bipolar regime until $\mu\left(r_{\text {bip }}\right) \approx \omega_{\mathrm{H}}$ 42]. At larger radii $\mu(r)<\omega_{\mathrm{H}}$ and the neutrino self-interaction becomes negligible. For our chosen $\mu_{0}$, an excess $\nu_{e}$ flux of $25 \%$, and $\omega_{\mathrm{H}}=0.3 \mathrm{~km}^{-1}$, we find a synchronization and bipolar radius of $r_{\text {syn }} \simeq 100 \mathrm{~km}$ and $r_{\text {bip }} \simeq 330 \mathrm{~km}$, as indicated in Fig. 1 by dark and light vertical gray bands, respectively. One important consequence of this flavor transformation in the context of three neutrino flavors is its potential sensitivity to deviations of $\vartheta_{23}$ from maximal mixing. As pointed out in Ref. [53], in the particular case that the $\mu \tau$-resonance takes place outside the bipolar radius, $r_{\mu \tau} \gtrsim r_{\text {bip }}$, the final states $\nu_{x} \bar{\nu}_{x}$, and therefore the final $\nu_{e}$ and $\bar{\nu}_{e}$ survival probability, depend crucially on the octant of $\vartheta_{23}$. According to Eq. (15) and the definition of $r_{\text {bip }}$ this condition is satisfied when

$\lambda_{0} Y_{\tau, \text { nsi }}^{\text {eff }} \gtrsim \omega_{\mathrm{H}}\left(\frac{r_{\text {bip }}}{R}\right)^{3}=\left[\left(\frac{\mu_{0}}{2}\right)^{3} \omega_{\mathrm{H}}\right]^{1 / 4} \sim 1.1 \times 10^{4} \mathrm{~km}^{-1}$

In the standard case the possibility to discern the $\vartheta_{23}$ octant only occurs for large density profiles, $\lambda_{0} \gtrsim 7 \times$ $10^{8} \mathrm{~km}^{-1}$, i.e. at early times. This situation would correspond to the $\lambda_{1}(r)$ profile in top panel of Fig. 1, but not to $\lambda_{2}(r)$. However the presence of NSI terms in the Hamiltonian may shift the $\mu \tau$-resonance to outer layers, making this condition more flexible. For instance, for $\varepsilon_{\tau \tau}=0.1$ the previous condition requires only $\lambda_{0} \gtrsim 1.3 \times 10^{5} \mathrm{~km}^{-1}$, see lower green band in the top panel of Fig. 1. Therefore the presence of NSI could keep the possibility to distinguish between the two $\vartheta_{23}$ octants for several seconds.

The self-induced flavor transformations however do not occur for arbitrarily large density profiles. If the electron density $n_{e}$ significantly exceeds the neutrino density $n_{\nu}$ in the conversion region they can be suppressed by matter [81]. This is a consequence of neutrinos traveling on different trajectories when streaming from a source that is not point-like. This multi-angle matter effect can be neglected if in the collective region, prior to the synchronization radius, we have

$$
\lambda^{\star}(r) \equiv Y_{e}(r) \lambda(r) \frac{R^{2}}{2 r^{2}} \ll \mu(r) .
$$

The limiting condition can be determined by imposing Eq. (20) at $r_{\text {syn }}$. Taking into account Eqs. (5) and (13) we obtain

$$
Y_{e}\left(r_{\mathrm{syn}}\right) \lambda_{0} \frac{R}{r_{\mathrm{syn}}} \ll \mu_{0} .
$$

If we assume $Y_{e}=0.5$ and $r_{\mathrm{syn}}=100 \mathrm{~km}$ then this condition amounts to $\lambda_{0} \ll 1.4 \times 10^{7} \mathrm{~km}^{-1}$. In the examples considered in the top panel of Fig. 1 this is fulfilled for $\lambda_{2,0}$. The condition $\lambda_{2}^{\star}(r) \ll \mu(r)$ is then satisfied in the bipolar region. As a consequence collective effects are not matter suppressed. This is not the case of $\lambda_{1}(r)$. In the standard case the limiting $\lambda_{0}=1.4 \times 10^{7} \mathrm{~km}^{-1}$, above which multi-angle matter effects suppress the collective effects, is, though, smaller than the minimum $\lambda_{0}=7 \times 10^{8} \mathrm{~km}^{-1}$ required for the $\mu \tau$ effect to be important. The situation could drastically change in the presence of NSI. As previously discussed, non-zero NSI non-universal (diagonal) parameters could help moving the $\mu \tau$ resonance out of the $r_{\mathrm{syn}}$ even for $\lambda_{0}$ smaller than $1.4 \times 10^{7} \mathrm{~km}^{-1}$. The consequence is that large enough $\left|\varepsilon_{\tau \tau}\right|$ would make the neutrino propagation through the SN envelope highly sensitive to the $\vartheta_{23}$ octant.

\section{CLASSIFICATION OF REGIMES}

In this section we summarize all the information formerly introduced. Taking into account the conditions given in Eqs. (18), (19), (20), and (21) we can roughly identify four different regimes of the neutrino propagation depending on $\lambda_{0}$ and $\varepsilon_{\tau \tau}$. This scheme is displayed in Fig. 2 for $\epsilon=0.25$ and $\mu_{0}=7 \times 10^{5} \mathrm{~km}^{-1}$. It is important to notice that the size of the different regions depends on the particular values of $2 \epsilon$ and $\mu_{0}$. Nevertheless, unless kinematic decoherence between different angular modes is triggered [45], this schematic picture certainly holds.

To first approximation the four regions can be defined in terms of matter suppression (or not) of collective effects and presence (or not) of the internal $I$-resonance. Equation (21) is depicted as a horizontal solid line at $\lambda_{0}=1.4 \times 10^{7} \mathrm{~km}^{-1}$. For higher $\lambda_{0}$ matter suppresses collective effects whereas for smaller densities collective effects are present. For intermediate values, $\lambda^{\star}(r) \sim \mu(r)$, there would be a matter induced decoherence [81]. To 
make the discussion as simple as possible we will only consider the extreme cases. On the other hand the vertical dashed line at $\varepsilon_{\tau \tau}=10^{-2}$ indicates the presence (right) or absence (left) of the NSI-induced $I$-resonance. This simple scheme becomes further complicated if one

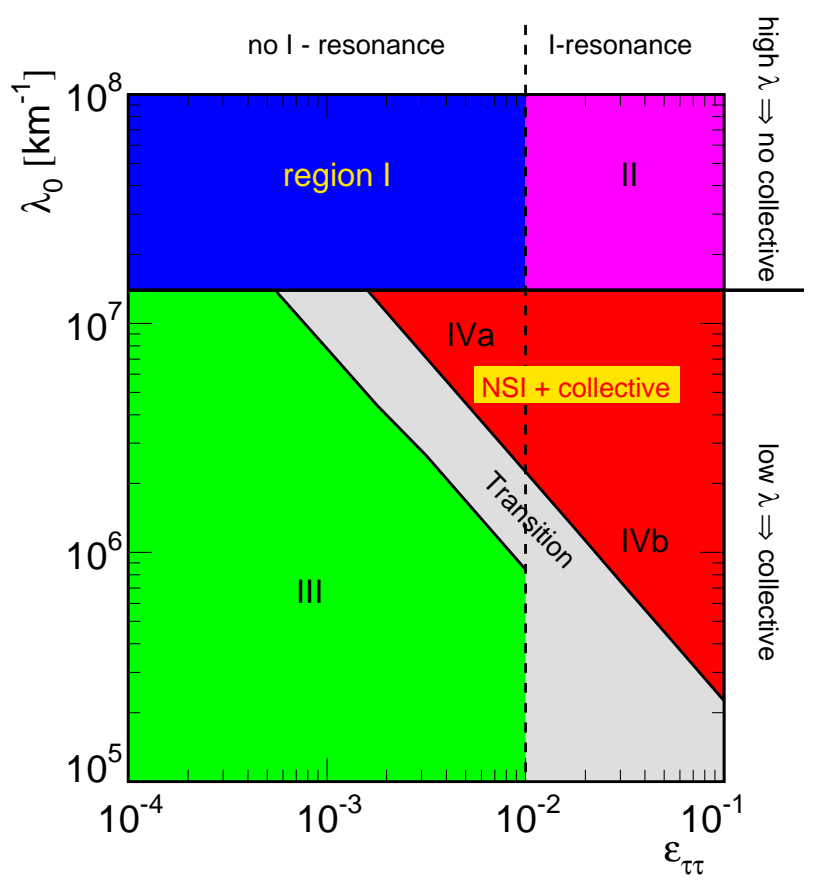

FIG. 2: Different regimes of the neutrino propagation depending on the value of $\lambda_{0}$ and $\varepsilon_{\tau \tau}$, as described in the text.

adds the possibility that the $\mu \tau$-resonance lies outside the bipolar region. In the next subsections we analyze in detail the different possibilities.

\section{A. Region I}

On the upper left corner we have the region I, defined by $\lambda_{0} \gtrsim 1.4 \times 10^{7} \mathrm{~km}^{-1}$ and $\varepsilon_{\tau \tau} \lesssim 10^{-2}$. According to the previous discussion, this range of parameters leads to no collective effects, since they are suppressed by matter, and no $I$-resonance. Assuming that the $L$ and $H$-resonances are adiabatic the $\nu_{e}$ and $\bar{\nu}_{e}$ survival probability is then only fixed by the mass hierarchy. The NSI terms will lead at most to a small shift in its position [36, 80].

In Fig. 3 we show the level crossing scheme for normal (top), and inverted mass hierarchy for $\vartheta_{23}<\pi / 4$ (middle), and $\vartheta_{23}>\pi / 4$ (bottom). It is remarkable to note the presence of a dip in the uppermost curves at high densities, corresponding to the $V_{e e}$ entry in Eq. (11). This is a direct consequence of the radial dependence of $Y_{e}(r)$, as given in Eq. (6). The arrows in these figures represent the transitions caused by the collective effects, and must therefore be ignored when these are not present. In the normal hierarchy case $\nu_{e}$ and $\bar{\nu}_{e}$ leave the SN as $\nu_{3}$ and $\bar{\nu}_{1}$, whereas for inverted mass hierarchy they escape as $\nu_{2}$ and $\bar{\nu}_{3}$ for any octant. The survival probabilities can then be written as $P\left(\nu_{e} \rightarrow \nu_{e}\right) \approx \sin ^{2} \vartheta_{13}\left(\sin ^{2} \vartheta_{12}\right)$ and $P\left(\bar{\nu}_{e} \rightarrow \bar{\nu}_{e}\right)=\cos ^{2} \vartheta_{12}\left(\sin ^{2} \vartheta_{13}\right)$ for normal (inverted) mass hierarchy. Figure 4 represents in solid lines the radial evolution of $\rho_{e e}$ and $\bar{\rho}_{e e}$ assuming $\lambda_{0}=1 \times 10^{8} \mathrm{~km}^{-1}$, $\omega_{\mathrm{H}}=0.3 \mathrm{~km}^{-1}, \sin ^{2} \vartheta_{23}=0.5$, and $\epsilon=0.25$. The vertical bands indicate where the resonance conversions take place. In order to perform the plot we have artificially set $\mu_{0}=0$. We want to remind here that both $\rho_{e e}$ and $\bar{\rho}_{e e}$ are normalized to the $\bar{\nu}_{e}$ flux, and therefore, while $\bar{\rho}_{e e}$ corresponds directly to $\bar{\nu}_{e}$ survival probability, $\rho_{e e}$ must be corrected by a factor $(1+\epsilon)$ in order to obtain the corresponding survival probability, $\rho_{e e}=P\left(\nu_{e} \rightarrow \nu_{e}\right)(1+\epsilon)$.

\section{B. Region II}

The region II, on the upper right corner, is defined by $\lambda_{0} \gtrsim 1.4 \times 10^{7} \mathrm{~km}^{-1}$ and $\varepsilon_{\tau \tau} \gtrsim 10^{-2}$. As in region I the matter density is so high that prevents neutrinos from undergoing collective effects. However, the values of the diagonal NSI terms in this region are large enough to fulfill Eq. (18), causing the $I$-resonance to appear. In contrast to the previous case $\nu_{e}$ and $\bar{\nu}_{e}$ are now created as $\nu_{2}^{\mathrm{m}}\left(\nu_{1}^{\mathrm{m}}\right)$ and $\bar{\nu}_{2}^{\mathrm{m}}\left(\bar{\nu}_{1}^{\mathrm{m}}\right)$ for normal (inverted) mass hierarchy ${ }^{5}$. They cross adiabatically all resonances and leave the SN as $\nu_{2}\left(\nu_{1}\right)$ and $\bar{\nu}_{2}\left(\bar{\nu}_{1}\right)$ for normal (inverted) mass hierarchy [36]. In Fig. 5 we show the level crossing scheme analogous to Fig. 3 but in the presence of the $I$-resonance. The survival probabilities are now

\footnotetext{
5 The different neutrino basis used in the analysis are: $\nu_{\alpha}$ with $\alpha=$ $e, \mu, \tau$ representing the flavor basis; $\nu_{i}$ with $i=1,2,3$ standing for the mass basis, diagonalizing the Hamiltonian in vacuum, $H=\Omega$; the matter basis, $\nu_{i}^{\mathrm{m}}$, diagonalizing the Hamiltonian in absence of neutrino self-interactions $H=\Omega+\mathrm{V}$; and the basis $\nu_{i}^{\prime}$ defined as the one diagonalizing the Hamiltonian in vacuum in the case that all mixing angles are very small (after rotating the matter term away).
} 

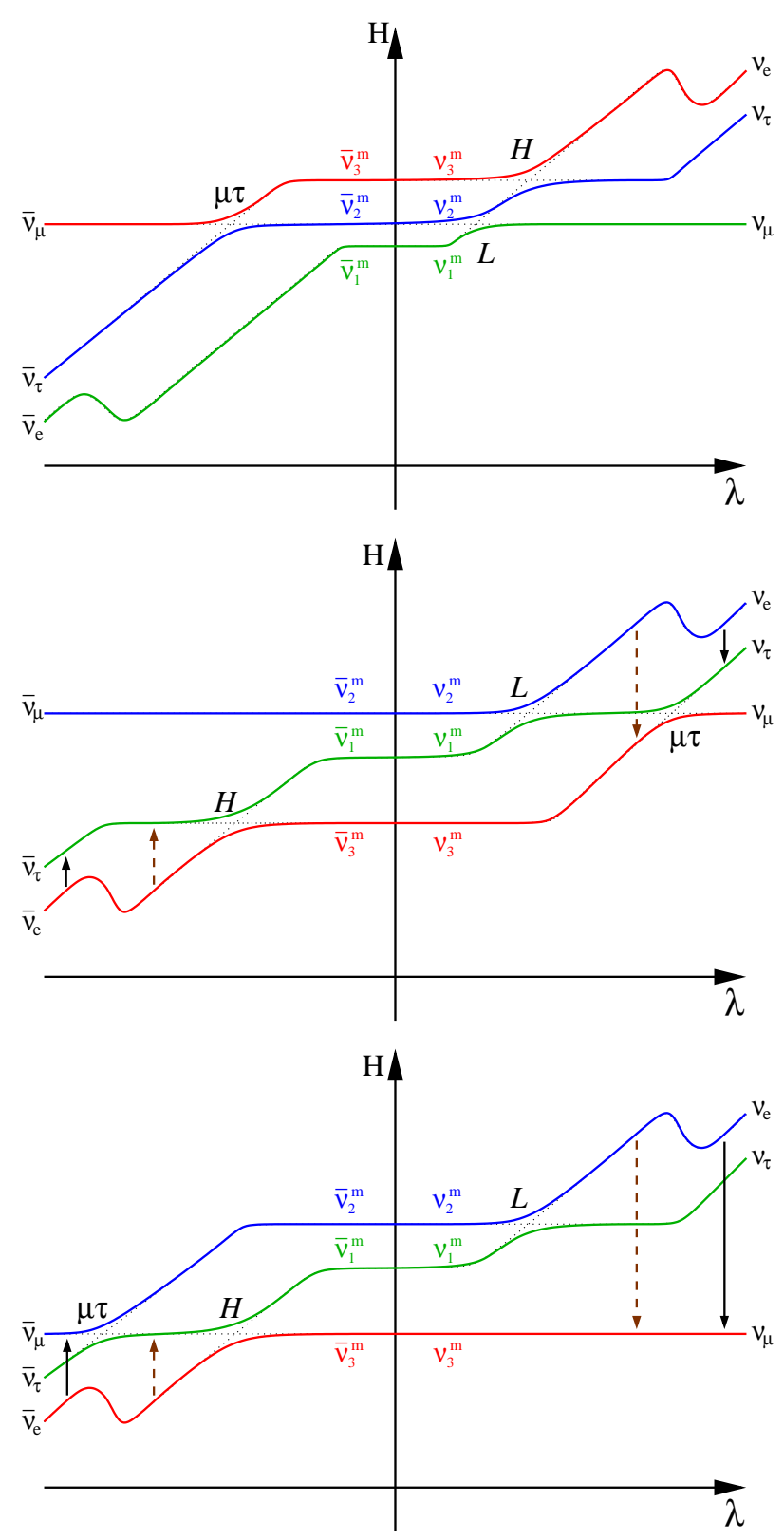

FIG. 3: Level crossings in the absence of $I$-resonance for normal and $\vartheta_{23}<\pi / 4$ (top), and inverted mass hierarchy for $\vartheta_{23}<\pi / 4$ (middle), and $\vartheta_{23}>\pi / 4$ (bottom). The dashed and solid arrows in the middle and bottom indicate the pair transformations due to collective effects happening after (dashed) or before (solid) the $\mu \tau$-resonance.

$P\left(\nu_{e} \rightarrow \nu_{e}\right)=P\left(\bar{\nu}_{e} \rightarrow \bar{\nu}_{e}\right) \approx \sin ^{2} \vartheta_{12}\left(\cos ^{2} \vartheta_{12}\right)$ for normal (inverted) mass hierarchy. The black dashed lines in Fig. 4 show the expected radial evolution of $\rho_{e e}$ and $\bar{\rho}_{e e}$, respectively, when neutrinos and antineutrinos undergo an adiabatic $I$-resonance. The width of the green band
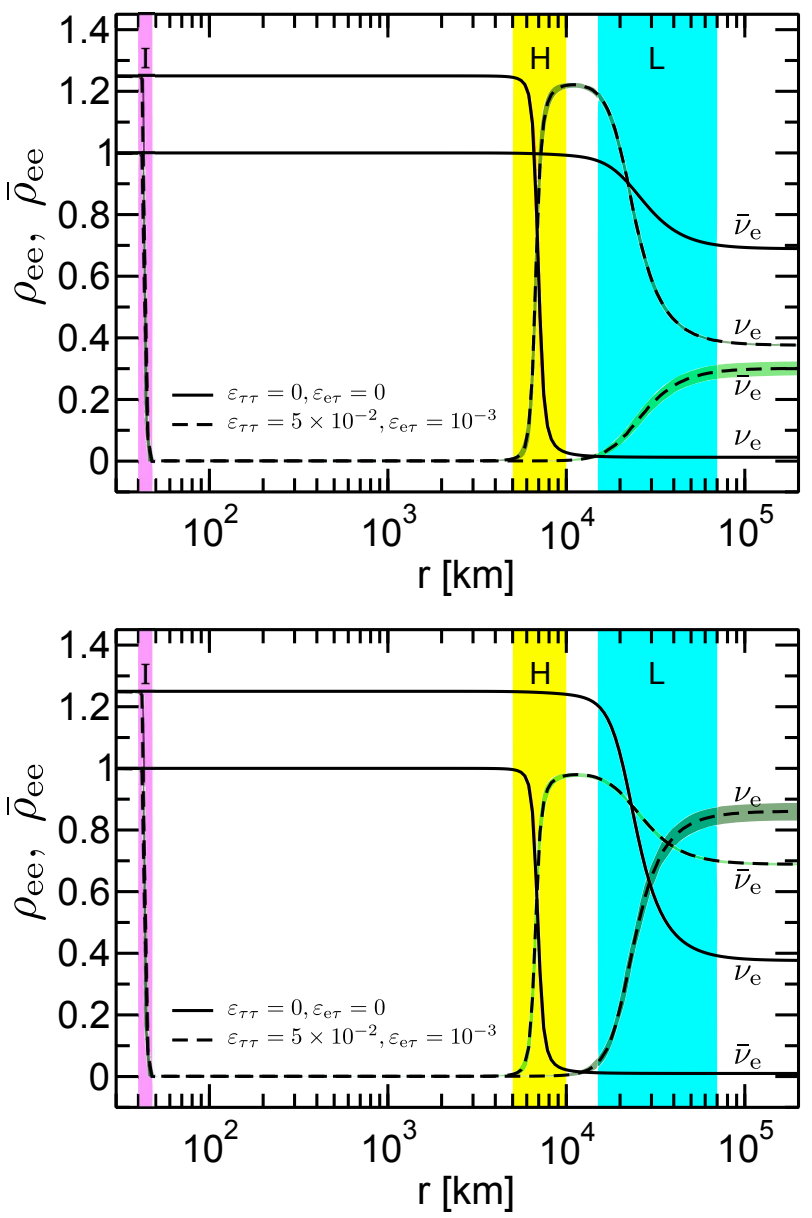

FIG. 4: Radial dependence of density matrix elements $\rho_{e e}$ and $\bar{\rho}_{e e}$ corresponding to regions I (solid) and II (dashed) in Fig. 2 Top panel represents normal mass hierarchy and bottom panel inverted mass hierarchy. We assume $\lambda_{0}=1 \times 10^{8} \mathrm{~km}^{-1}$, $\omega_{\mathrm{H}}=0.3 \mathrm{~km}^{-1}$, and $\sin ^{2} \vartheta_{23}=0.5$. Vertical bands indicate regions where resonances take place.

accounts for the oscillatory behavior of neutrinos. As for region I, we have made the calculation assuming $\mu_{0}=0$. However, we have analyzed the single energy and multiangle case within two-flavor framework for the range of parameters here discussed, and have verified that collective effects are indeed suppressed and the $I$-resonance is present for both normal and inverted hierarchies. This means that the behavior in region II corresponds indeed to the case discussed in Ref. [36]. 

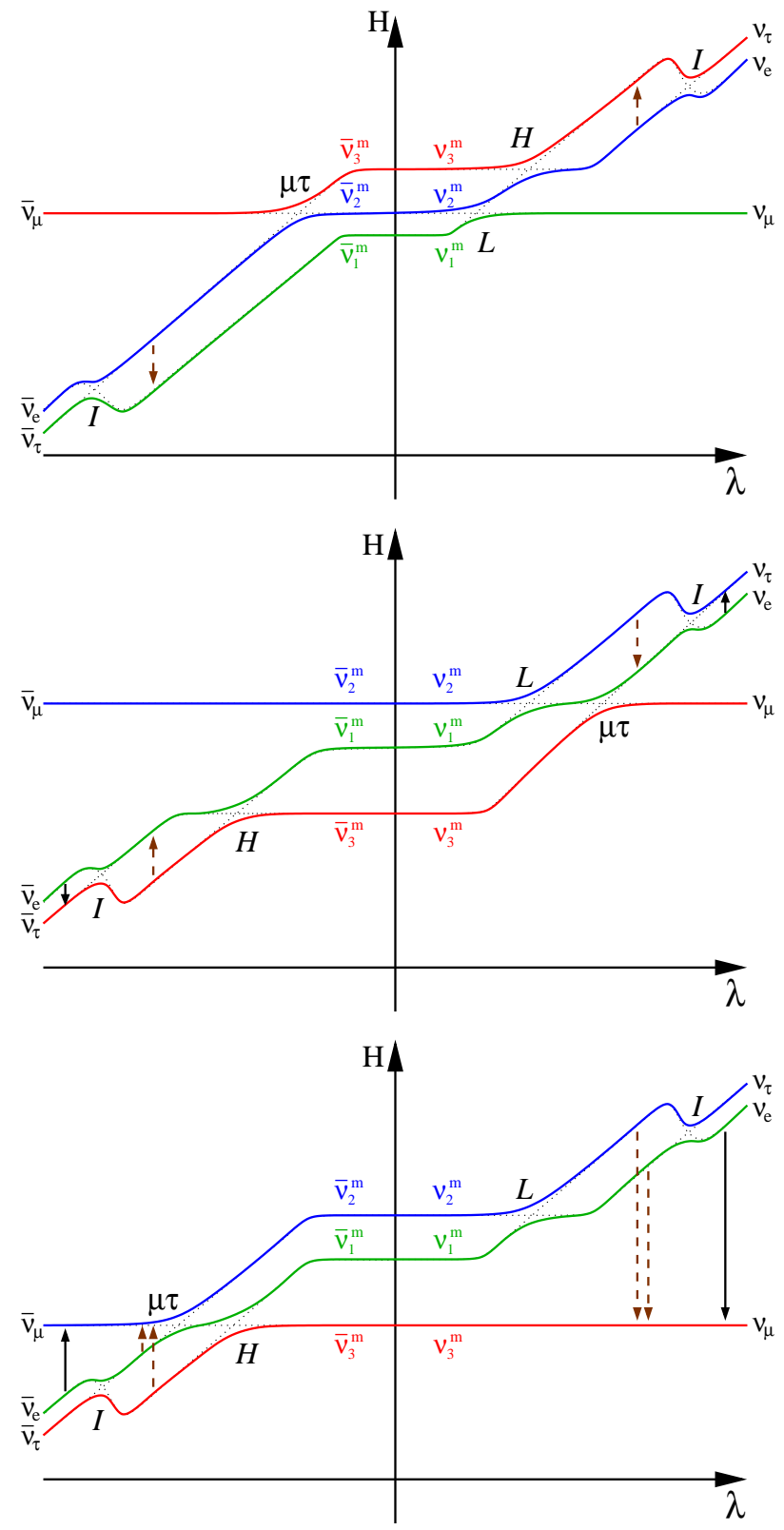

FIG. 5: Same as Fig. 3in the presence of $I$-resonance. Dashed arrows represent pair transformations due to collective effects occurring after the $I$-resonance. In the bottom panel there are two possibilities for this transition, corresponding to an adiabatic or non-adiabatic $I$-resonance. The solid arrows in the middle and bottom panel refer to collective effects happening before the $I$-resonance.

\section{Region III}

Let us now consider the lower part of Fig. 2, i.e. when $\lambda_{0} \lesssim 1.4 \times 10^{7} \mathrm{~km}^{-1}$. The main feature of this scenario is the presence of collective effects. As discussed in
Ref. 53], and here reviewed, these in turn depend on the relative position of the $\mu \tau$-resonance with respect to the synchronization and bipolar radius. We can then distinguish two different regimes: On the bottom left corner we define region III by the condition $r_{\mu \tau} \lesssim r_{\text {bip }}$, and on the bottom right corner we have region IV defined by $r_{\mu \tau} \gtrsim r_{\text {bip }}$. In the middle of both there is a transition region whose width we have determined numerically.

Let us now discuss region III. According to Eq. (19) this range of parameters satisfies the condition

$$
\lambda_{0}\left[Y_{\tau}^{\mathrm{eff}}+\left(2-Y_{e}\right) \varepsilon_{\tau \tau}\right] \lesssim 1.1 \times 10^{4} \mathrm{~km}^{-1},
$$

which, for $Y_{e}=0.5$, roughly amounts to $\lambda_{0} \varepsilon_{\tau \tau} \lesssim$ $7.3 \times 10^{3} \mathrm{~km}^{-1}$, see Fig. 2, This situation can be reduced to the standard two-flavor scenario. In order to better understand the consequences of collective effects it is convenient to realize that the impact of ordinary matter can be transformed away by going into a rotating reference frame for the polarization vectors [40, 42]. Collective conversions proceed in the same way as they would in the absence of the matter term $V_{\text {std }}$, except that the effective mixing angle is reduced. After this rotation the mass eigenstates $\nu_{i}^{\prime}$ now approximately coincide with the interaction eigenstates $\nu_{\alpha}$. In particular, the initial states $\nu_{e}$ and $\bar{\nu}_{e}$ can therefore be essentially identified with $\nu_{1}^{\prime}$ and $\bar{\nu}_{1}^{\prime}$, respectively. If the neutrino mass hierarchy is normal, we begin in the lowest-lying state and no collective effects take place, see bottom left panel of Fig. 6 (also in Fig. 4 of Ref. [53]). The situation is then similar to that in region I, see top panel of Fig. 4. However in the case of inverted mass hierarchy both $\nu_{1}^{\prime}$ and $\bar{\nu}_{1}^{\prime}$ correspond to the intermediate state. The effect of the self-interaction is to drive them to the lowest-lying states, which in this case are $\nu_{3}^{\prime}$ and $\bar{\nu}_{3}^{\prime}$, see top left panel of Fig. 6. In terms of matter eigenstates $\nu_{i}^{\mathrm{m}}$ this is shown by dashed arrows in the middle and bottom panels of Fig. 3. In the case of $\nu_{e}$ a fraction equal to $\epsilon F_{\bar{\nu}_{e}}$ is not transformed and stays in $\nu_{2}^{\mathrm{m}}$ and evolves as in the absence of neutrino-neutrino interactions, i.e. adiabatically through the $L$-resonance. The rest of $\nu_{e}$ are transformed to $\nu_{3}^{\mathrm{m}}$. As a result the final $\nu_{e}$ flux, normalized to the initial $\bar{\nu}_{e}$ flux, is expected to be approximately $\rho_{e e}^{\text {final }}=\epsilon \sin ^{2} \vartheta_{12}+\sin ^{2} \vartheta_{13} \simeq 0.08$. On the other hand, after the pair transformation $\bar{\nu}_{e}$ cross the $H$-resonance adiabatically and leave the star as $\nu_{1}$, leading to a final 

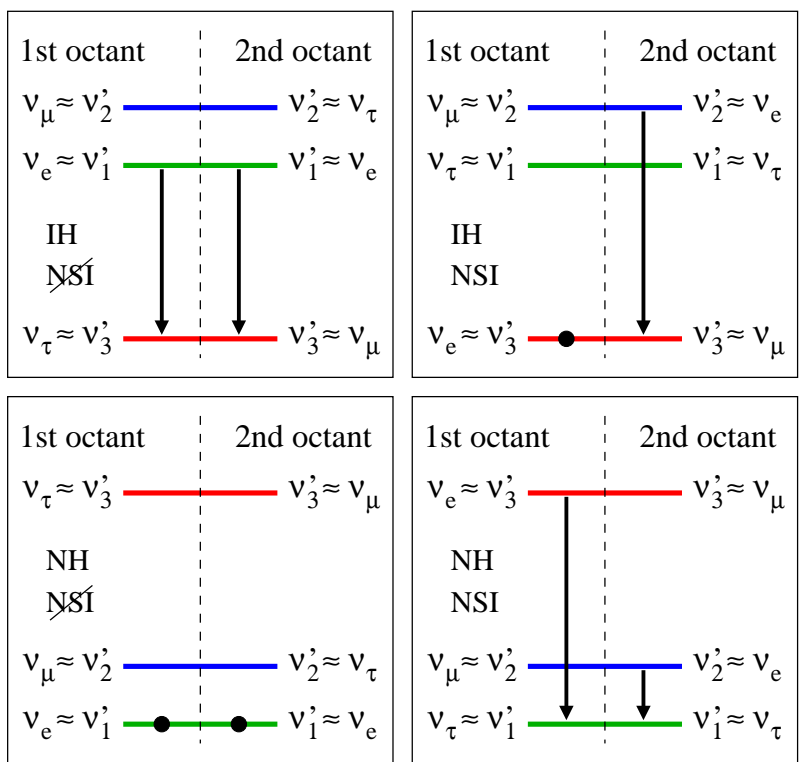

FIG. 6: Neutrino vacuum level diagrams without NSI effects (left panels) and with the NSI-induced $I$ resonance occurring before the bipolar conversion (right panels). The top (bottom) panels correspond to inverted (normal) neutrino mass hierarchy. In each panel the two possible $\vartheta_{23}$ are indicated. The 12 and 13-mixing angles are assumed to be very small, mimicking the effect of ordinary matter. The effect of collective conversions is indicated by an arrow.

normalized flux of approximately $\bar{\rho}_{e e}^{\text {final }}=\cos ^{2} \vartheta_{12} \simeq 0.7$. This can be seen in Fig. 7, where we show in solid lines the radial evolution of $\nu_{e}$ and $\bar{\nu}_{e}$ for inverted mass hierarchy assuming $\lambda_{0}=4 \times 10^{6} \mathrm{~km}^{-1}, \omega_{\mathrm{H}}=0.3 \mathrm{~km}^{-1}$, and $\sin ^{2} \vartheta_{23}=0.4$ (top) and 0.6 (bottom). As can be seen in the figure, the result is independent of the $\vartheta_{23}$ octant.

\section{Region IV}

Finally, neutrinos with parameters in the right bottom corner (region IV) will feel both collective and NSI effects. This region of parameters is defined by the condition that the $\mu \tau$-resonance lies outside the bipolar region. According to Eq. (19) this amounts to

$$
\lambda_{0}\left[Y_{\tau}^{\mathrm{eff}}+\left(2-Y_{e}\right) \varepsilon_{\tau \tau}\right] \gtrsim 1.1 \times 10^{4} \mathrm{~km}^{-1} .
$$

As discussed above, for the standard case and $Y_{e}=0.5$ this is satisfied for $\lambda_{0} \gtrsim 7 \times 10^{8} \mathrm{~km}^{-1}$, which implies a strong matter suppression of the collective effects, see Fig. 2. However, if NSI diagonal parameters are of the
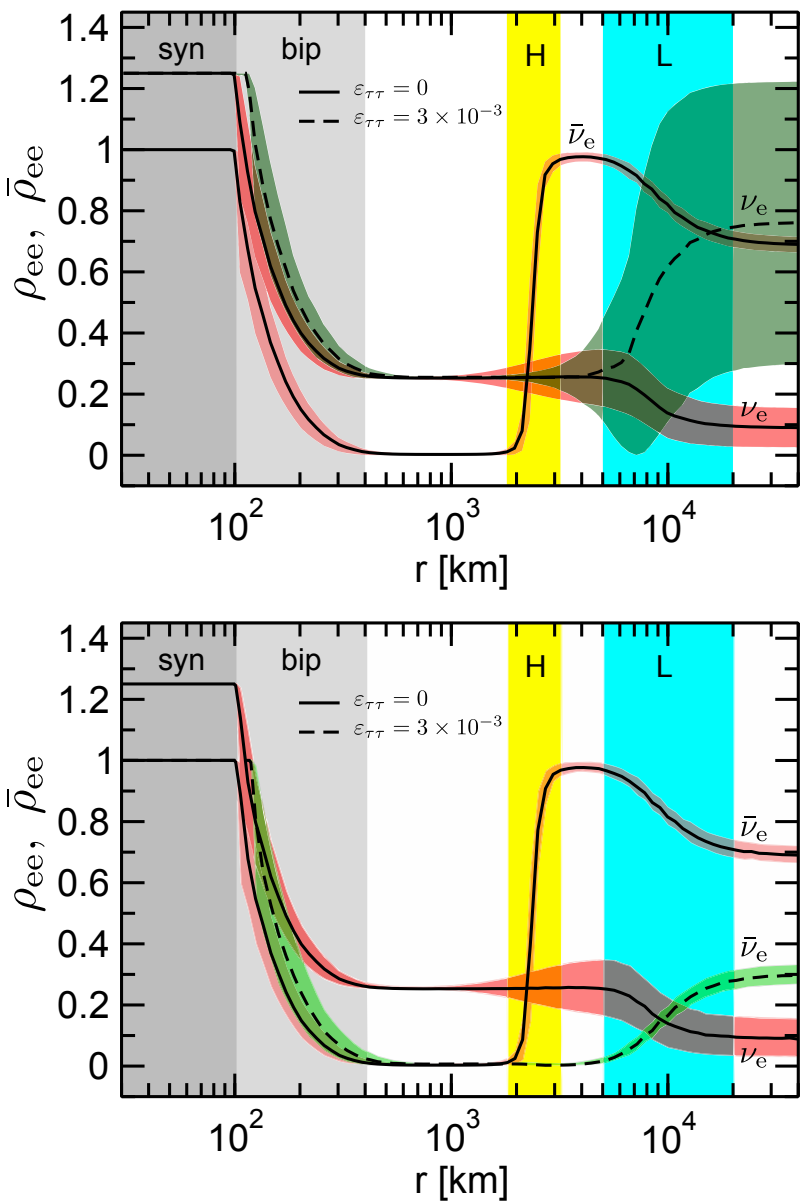

FIG. 7: Radial dependence of $\rho_{e e}$ and $\bar{\rho}_{e e}$ for region III with $\varepsilon_{\tau \tau}=0$ (solid) and IVa (dashed) with $\varepsilon_{\tau \tau}=3 \times 10^{-3}$ for inverted mass hierarchy, and $\sin ^{2} \vartheta_{23}=0.4(0.6)$ in the top (bottom panel). In both cases $\lambda_{0}=4 \times 10^{6} \mathrm{~km}^{-1}$ and $\varepsilon_{e \tau}=0$. The bands around the lines represent modulations. Vertical gray bands stand for synchronized (dark) and bipolar (light) regime. Resonance regions are also displayed.

order of $\left|\varepsilon_{\tau \tau}\right| \gtrsim 7.3 \times 10^{3} / \lambda_{0}\left(\mathrm{~km}^{-1}\right)$ then one can avoid the matter suppression condition. Therefore the first NSI effect is to increase the value of $Y_{\tau, \text { nsi }}^{\text {eff }}$ so that the $\lambda_{0}$ required to have the $\mu \tau$-resonance outside $r_{\text {bip }}$ is still consistent with the presence of collective effects. Moreover, if $\varepsilon_{\tau \tau}$ is of the order of a few $\%$ the condition given in Eq. (18) is fulfilled for the typical values of $Y_{e}$ found in SNe. Thus, in the region IV we can distinguish two subsets of parameters denoted by IVa and IVb defined by the absence or presence of the $I$-resonance, respectively. For defineteness we set the boundary at $\varepsilon_{\tau \tau}=10^{-2}$.

Let us first consider the IVa region. Depending on $\lambda_{0}$, 
i.e. on the instant considered, this range of parameters implies values of $\left|\varepsilon_{\tau \tau}\right|$ from $10^{-3}$ to $\sim 10^{-2}$. Although values are not high enough to induce the $I$ resonance they are sufficiently large to push the $\mu \tau$-resonance outside the bipolar region. The situation is therefore analogous to the one described in Ref. [53]. That means a flavor pair transformation $\nu_{e} \bar{\nu}_{e} \rightarrow \nu_{x} \bar{\nu}_{x}$ due to collective effects only for inverted neutrino mass hierarchy, like in region III. However, the final matter eigenstates depend on the $\vartheta_{23}$ octant. In the middle panel of Fig. 3 we show with solid lines the pair conversion for $\vartheta_{23}$ in the first octant. In terms of matter eigenstates, $\nu_{e}$ and $\bar{\nu}_{e}$ are transformed into $\nu_{1}^{\mathrm{m}}$ and $\bar{\nu}_{1}^{\mathrm{m}}$, respectively. The presence of the $\mu \tau$ resonance in the neutrino channel leads to a difference of $\nu_{e}$ with respect to region III. In the top panel of Fig. 7 we show with dashed lines the evolution of $\nu_{e}$ as function of the distance, for $\varepsilon_{\tau \tau}=3 \times 10^{-3}$ and $\sin ^{2} \vartheta_{23}=0.4$. In the collective bipolar conversions, the excess $\epsilon$ of $\nu_{e}$ over $\bar{\nu}_{e}$ remains as $\nu_{2}^{\mathrm{m}}$ whereas the rest will be transformed to $\nu_{1}^{\mathrm{m}}$. As a consequence, the original $\nu_{e}$ flux leaving the star can be written as $\rho_{e e}^{\text {final }}=\varepsilon \sin ^{2} \vartheta_{12}+\cos ^{2} \vartheta_{12}$, which in our particular case amounts to roughly 0.75 . If $\vartheta_{23}$ belongs to the second octant the $\mu \tau$-resonance takes place in the antineutrino channel, see bottom panel of Fig. 3. The pair $\nu_{e}$ and $\bar{\nu}_{e}$ is driven to the lowest-lying states, which in this case are $\nu_{3}^{\mathrm{m}}$ and $\bar{\nu}_{2}^{\mathrm{m}}$, for neutrinos and antineutrinos, respectively. Therefore, for $\nu_{e}$ the situation is completely analogous to that in region III, whereas $\bar{\nu}_{e}$ leave the star as $\bar{\nu}_{2}$. The radial evolution of $\bar{\nu}_{e}$ for $\varepsilon_{\tau \tau}=3 \times 10^{-3}$ and $\sin ^{2} \vartheta_{23}=0.6$ is displayed with dashed lines in the bottom panel of Fig. 7 It is remarkable that neither $\nu_{e}$ nor $\bar{\nu}_{e}$ undergo the $\mathrm{H}$ resonance, and therefore are blind to the possible effect of the outwards propagating shock wave 82 84].

It is important to notice that the same effect observed for the different octants of $\vartheta_{23}$ can be obtained by fixing the octant and changing the sign of $\varepsilon_{\tau \tau}$. This can be easily understood if we study the $\mu \tau$-resonance condition,

$$
\lambda(r)\left[Y_{\tau}^{\mathrm{eff}}+\left(2-Y_{e}\right) \varepsilon_{\tau \tau}\right] \simeq-\omega_{\mathrm{H}} \cos ^{2} \vartheta_{13} \cos 2 \vartheta_{23}
$$

where we have neglected subleading solar terms. This condition dictates the channel where the resonance takes place. In the standard case this is determined only by the hierarchy of neutrino masses and the octant of $\vartheta_{23}$. In the presence of NSI, though, the sign of left-hand side of the equation depends on that of $\varepsilon_{\tau \tau}$, what therefore affects directly the resonance condition. As a result, the same result of Fig. 7 is obtained by changing the sign of $\varepsilon_{\tau \tau}$ and the octant of $\vartheta_{23}$, i.e. $\varepsilon_{\tau \tau}=-3 \times 10^{-3}$ and second octant for the panel on top and first octant in the bottom panel. In summary, in the presence of nonuniversal NSI parameters with $\left|\varepsilon_{\tau \tau}\right| \gtrsim 10^{-3}$, SN neutrinos are sensitive to the octant of $\vartheta_{23}$, the absolute value of the non-universal NSI parameter as well as its sign.

Finally, for higher values of the non-universal NSI parameters, $\varepsilon_{\tau \tau} \gtrsim 10^{-2}$ (region IVb), the internal $I$ resonance will arise. In this case one must analyze the interplay between collective effects and the $I$-resonance. This is discussed in the next section.

\section{COLLECTIVE EFFECTS AND NSI-INDUCED I-RESONANCE}

In this section we analyze region IVb, defined by $\lambda_{0} \lesssim$ $1.4 \times 10^{7} \mathrm{~km}^{-1}$ and $\varepsilon_{\tau \tau} \gtrsim 10^{-2}$, where both an adiabatic $I$-resonance and collective effects are present. If the $I$ resonance is not adiabatic then neutrinos within region $\mathrm{IVb}$ evolve exactly as in IVa.

As can be inferred from Fig. 1, one of the main features of this scenario is that both effects happen nearly in the same region, namely the deepest layers right above the neutrinosphere. This means that the final result will also depend on the relative position between the bipolar region and the location of the $I$-resonance. Schematically two extreme scenarios can be identified. In one case the rise in the $Y_{e}$, and consequently the $I$-resonance, takes place before the bipolar conversion region, see $Y_{e}^{\mathrm{a}}$ in the bottom panel of Fig. 1. In the second scenario one has first the bipolar conversion and then neutrinos traverse the $I$-resonance, see $Y_{e}^{\mathrm{b}}$ in the bottom panel of Fig. 1].

\section{A. First NSI $I$-resonance}

Let us analyze here the particular case where the $I$ resonance happens in deeper layers than collective effects. This situation corresponds to the $Y_{e}^{\mathrm{a}}$ in the bottom panel of Fig. 1] and, according to SN numerical simulations it is the most likely situation.

The main consequence of an $I$-resonance in the inner- 
most layers, right after the neutrinosphere, is an inversion of the neutrino fluxes entering the bipolar region. For the initial flux pattern assumed in Sec. Ithis implies the following new pattern after the $I$-resonance: $F_{\nu_{e}}=F_{\bar{\nu}_{e}}=0$, $F_{\nu_{\tau}}=1+\epsilon$, and $F_{\bar{\nu}_{\tau}}=1$ normalized to $F_{\bar{\nu}_{e}}$. In contrast to the standard case, under this condition collective effects arise in the case of normal mass hierarchy.

This can be understood using the pendulum analogy [42] in the corresponding reduced two flavor scenario, and keeping in mind that the bipolar conversion drives the neutrinos to the lowest-lying states. In the normal hierarchy the system is already created near the minimum of the potential. Thus, in absence of the $I$-resonance collective effects are not present. However once an adiabatic $I$-resonance occurs the neutrino flavor is swapped and the system is driven to the maximum of the potential. The original $\nu_{e}\left(\bar{\nu}_{e}\right)$ are transformed to $\nu_{\tau}\left(\bar{\nu}_{\tau}\right)$ and, in terms of the vacuum level diagrams, occupy now the highest states right before collective effects are switched on. In the right panels of Fig. 6 we show the vacuum level diagrams after an adiabatic $I$-resonance has occurred. In the case of normal mass hierarchy (bottom right panel) bipolar effects act taking $\nu_{e}\left(\bar{\nu}_{e}\right)$ to the lowest-lying states $\nu_{1}^{\prime}\left(\bar{\nu}_{1}^{\prime}\right)$, which in this case do not depend on the $\vartheta_{23}$ octant. This bipolar conversion can be also seen in term of matter eigenstates as dashed arrows in the top panel of Fig. 5. As a result both the $I$-resonance and the induced collective effects basically cancel each other. In the top panel of Fig. 8 we show the radial evolution of $\nu_{e}$ and $\bar{\nu}_{e}$. This cancellation between the $I$-resonance and collective effects is complete for $\bar{\nu}_{e}$, which leave as $\bar{\nu}_{1}$, but not for $\nu_{e}$ : its excess $\epsilon$ over $\bar{\nu}_{e}$ remains as $\nu_{3}\left(\nu_{2}\right)$ for $\vartheta_{23}$ in the first (second) octant. Therefore for a monochromatic neutrino flux we obtain, after propagating through the outer resonances, $\rho_{e e}^{\text {final }}=\epsilon \sin ^{2} \vartheta_{12}+\sin ^{2} \vartheta_{13} \approx 0.08$ for both $\vartheta_{23}$ octants, see top panel of Fig. 8, instead of simply $\sin ^{2} \vartheta_{13}=10^{-2}$ as in top panel of Fig. 4. Hence, by comparing the top panels of Figs. 4 and 8 one realizes that, except for the excess $\epsilon$, the situation for normal mass hierarchy is basically the same as in regions I, III, and IVa.

While this is true in the monoenergetic case a specific signature can be observed if we do not restrict ourselves to that case but consider the whole energy spectrum. The top panel of Fig. 9 displays the $\nu_{e}$ and $\nu_{x}$ fluxes at the neutrinosphere, $f_{\nu_{e}}^{\mathrm{R}}$ and $f_{\nu_{\vartheta}}^{\mathrm{R}}$. We have assumed the parameterization given in Ref. [85],

$$
f_{\nu_{\alpha}}^{R}(E)=C_{\nu_{\alpha}}\left(\frac{E}{\left\langle E_{\nu_{\alpha}}\right\rangle}\right)^{\beta_{\nu_{\alpha}}-1} \exp \left(-\beta_{\nu_{\alpha}} \frac{E}{\left\langle E_{\nu_{\alpha}}\right\rangle}\right)
$$

with $\left\langle E_{\nu_{e}}\right\rangle=12 \mathrm{MeV},\left\langle E_{\nu_{e}}\right\rangle=15 \mathrm{MeV},\left\langle E_{\nu_{x}}\right\rangle=18 \mathrm{MeV}$, $\beta_{\nu_{e}}=5, \beta_{\bar{\nu}_{e}}=4.5$ and $\beta_{\nu_{x}}=4$. The normalization $C_{\nu_{\alpha}}$ has been chosen such that $F_{\bar{\nu}_{e}}^{\mathrm{R}} \equiv \int f_{\bar{\nu}_{e}}^{\mathrm{R}}(E) \mathrm{d} E=1$, $F_{\nu_{e}}^{\mathrm{R}}=1+\kappa \epsilon$ and $F_{\nu_{x}}^{\mathrm{R}}=1-\kappa$, with $\kappa=0.15$.

In the bottom panel of Fig. 9 we show the $\nu_{e}$ fluxes after the bipolar region. In solid dark red lines we represent the case of normal mass hierarchy in region IVb. By comparing the two panels one sees how the conversion $\nu_{e} \rightarrow \nu_{x}$ takes place only at low energies. This is exactly the opposite of what happens for the standard case (inverted mass hierarchy in region III), shown as solid light red lines, where the untransformed flux concentrates at low energies [46, 47]. For completeness we show also the other cases. In the region of parameters I, III and IVa there is neither collective effects nor $I$-resonance for normal hierarchy, then the fluxes after the bipolar region coincide with the initial ones, $f_{\nu_{e}}=f_{\nu_{e}}^{R}$ and $f_{\nu_{x}}=f_{\nu_{x}}^{R}$. In region II, the $I$-resonance implies a complete conversion $\nu_{e} \rightarrow \nu_{x}$, which leads to a spectral swap, $f_{\nu_{e}}=f_{\nu_{x}}^{R}$ and $f_{\nu_{x}}=f_{\nu_{e}}^{R}$.

The case of inverted mass hierarchy is more subtle. According to the previous discussion one would expect no collective effects after neutrinos traverse the $I$-resonance. The system starts its evolution near the maximum of the potential and, in the absence of NSI, the bipolar conversions would take it to the minimum. What the $I$ resonance is doing in this language by swapping the flavor eigenstates is to take the system to the minimum of the potential before any collective effects can arise. The new stable situation prevents bipolar conversions, leaving the system unchanged until the outer resonances are reached. And this is indeed what happens if $\vartheta_{23}$ lies in the first octant. In terms of the vacuum levels diagram the original $\nu_{e}\left(\bar{\nu}_{e}\right)$ are transformed after the $I$-resonance into $\nu_{\tau}$ $\left(\bar{\nu}_{\tau}\right)$, which are already the lowest-lying states, see the top right panel in Fig. [6] Hence no collective effects take place, and, as can be seen in the middle panel of Fig. 5 after traversing the outer resonances $\nu_{e}$ and $\bar{\nu}_{e}$ leave the star as $\nu_{1}$ and $\bar{\nu}_{1}$. The radial evolution is shown with solid lines in the bottom panel of Fig. 8 . 

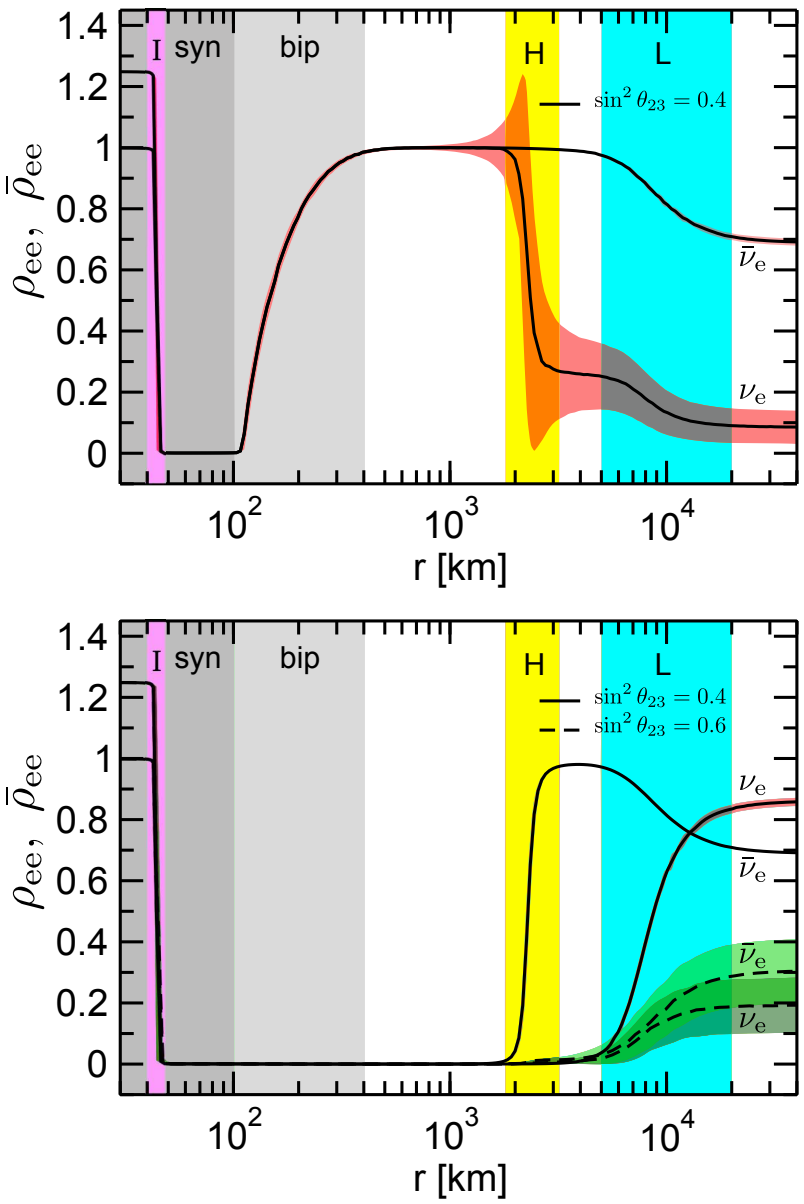

FIG. 8: Same as Fig. 4 for region IVb, for normal (top panel) and inverted (bottom panel) mass hierarchy. In the top panel it is shown the case $\sin ^{2} \vartheta_{23}=0.4$, while in the bottom panel the case $\sin ^{2} \vartheta_{23}=0.6$ is also displayed. In both cases $\lambda_{0}=$ $4 \times 10^{6} \mathrm{~km}^{-1}$ and $\varepsilon_{\tau \tau}=5 \times 10^{-2}$ and $\varepsilon_{e \tau}=10^{-3}$. The $I$-resonance is assumed to occur before the collective effects

However if $\vartheta_{23}$ lies in the second octant things are different. As in the previous case, the $I$-resonance converts neutrinos created initially as $\nu_{e}$ and $\bar{\nu}_{e}$ into $\nu_{\tau}$ and $\bar{\nu}_{\tau}$, respectively. But these states are now the highest states, $\nu_{2}^{\prime}$ and $\bar{\nu}_{2}^{\prime}$. This means, that in contrast to the first-octant case, when the neutrinos traverse the bipolar regime they will be driven to the lowest-lying states, i.e. $\nu_{2}^{\prime} \bar{\nu}_{2}^{\prime} \rightarrow \nu_{3}^{\prime} \bar{\nu}_{3}^{\prime}$, see top right panel of Fig. 6. In the bottom panel of Fig. 8 we show with dashed lines the evolution of $\nu_{e}$ and $\bar{\nu}_{e}$ as function of distance for $\sin ^{2} \vartheta_{23}=0.6$. The bipolar conversion can not be directly seen as it occurs between states containing explicitly neither $\nu_{e}$ nor $\bar{\nu}_{e}$. At the end, as shown in the bottom panel of Fig. 5 , the original $\bar{\nu}_{e}$
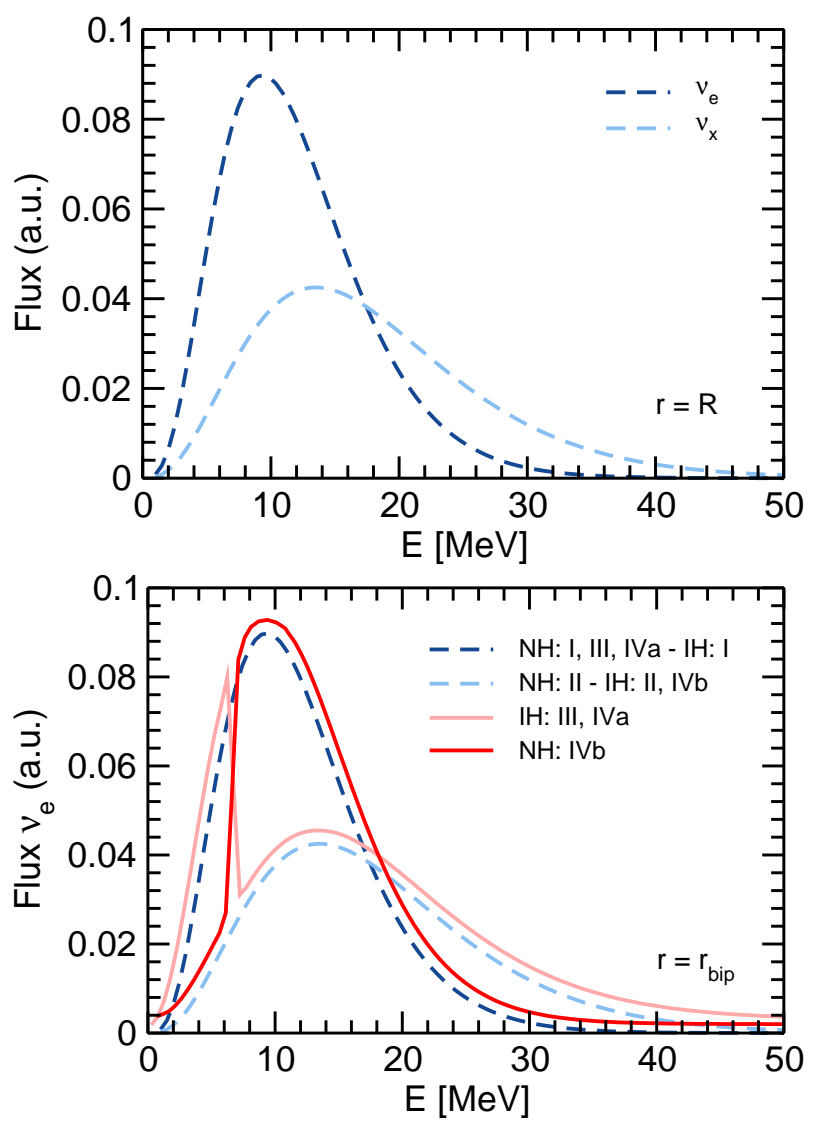

FIG. 9: Top: $\nu_{e}$ and $\nu_{x}$ fluxes as emitted at the neutrinosphere. Bottom: $\nu_{e}$ flux after the bipolar region for different cases. In the region IVb it is assumed that the $I$-resonance occurs before than the bipolar conversion. Note a depletion in the flux of low-energy neutrinos, instead of usual spectral split.

leave the $\mathrm{SN}$ as $\bar{\nu}_{2}$, see bottom panel of Fig. 8, In the case of $\nu_{e}$ the excess $\epsilon$ over $\bar{\nu}_{e}$ remains as $\nu_{2}$ whereas the rest is transformed to $\nu_{3}$. Therefore for a monoenergetic flux we find $\rho_{e e}^{\text {final }}=\epsilon \cos ^{2} \vartheta_{12}+\sin ^{2} \vartheta_{13} \approx 0.2$. By comparing the bottom panel of Figs. 8 and 7 one realizes that this case is analogous to IVa. Since the collective effects do not affect $\nu_{e}$ and $\bar{\nu}_{e}$ directly, considering neutrinos with an energy spectrum, one expects simply a complete swap of spectra, $f_{\nu_{e}}=f_{\nu_{x}}^{R}$ and $f_{\nu_{x}}=f_{\nu_{e}}^{R}$ after the bipolar region, like in scenario II, see bottom panel of Fig. 9

The final conclusion is that the propagation of neutrinos with parameters in region IV is practically independent of whether the $I$-resonance is present (IVb) or not (IVa). The main consequence of the $I$-resonance is to swap the $\nu_{e}$ spectrum at low energies and only in the 
case of normal mass hierarchy, in contrast to the transformation of high-energy $\nu_{e}$ happening for inverted mass hierarchy in the absence of $I$-resonance.

\section{B. First Collective}

For completeness we have also considered the possibility that the bipolar conversion takes place before neutrinos traverse the $I$-resonance. This situation corresponds schematically to the $Y_{e}^{\mathrm{b}}$ profile in the bottom panel of Fig. 1]

The case of normal mass hierarchy is completely analogous to the one of region II, that is, absence of collective effects and $I$-resonance. The $\nu_{e}$ and $\bar{\nu}_{e}$ are created as $\nu_{2}^{\mathrm{m}}$ and $\bar{\nu}_{2}^{\mathrm{m}}$, respectively. Therefore, if all resonances involved, $I, \mu \tau, H$, and $L$, are adiabatic then they leave the SN as $\nu_{2}$ and $\bar{\nu}_{2}$, respectively, see top panel of Fig. 10. The result is identical to the one shown with dashed lines in the top panel of Fig. 4

The situation with inverted mass hierarchy depends significantly on the $\vartheta_{23}$ octant. Rotating the matter term away $\nu_{e}$ and $\bar{\nu}_{e}$ are created as the intermediate states $\nu_{1}^{\prime}$ and $\bar{\nu}_{1}^{\prime}$. Collective effects drive them to the lowestlying states $\nu_{3}^{\prime}$ and $\bar{\nu}_{3}^{\prime}$. However the corresponding matter eigenstates are different depending on whether $\vartheta_{23}$ belongs to the first or to the second octant. In the first case, most of $\nu_{e}$ and $\bar{\nu}_{e}$ end up as $\nu_{2}^{\mathrm{m}}$ and $\bar{\nu}_{3}^{\mathrm{m}}$ before crossing the $I$-resonance, see solid arrows in the middle panel of Fig. 5. The excess $\epsilon$ of $\nu_{e}$ stays as $\nu_{1}^{\mathrm{m}}$. As a consequence, the final $\nu_{e}$ and $\bar{\nu}_{e}$ fluxes, normalized to the initial $\bar{\nu}_{e}$ one, are $\rho_{e e}^{\text {final }}=\epsilon \cos ^{2} \vartheta_{12}+\sin ^{2} \vartheta_{12}$ and $\bar{\rho}_{e e}^{\text {final }}=\sin ^{2} \vartheta_{13}$, respectively. See solid lines in the bottom panel of Fig. 10. Except for the excess $\epsilon$ in $\nu_{e}$ the net result is a cancellation of the collective effects and the $I$-resonance, leading to a similar result as in region I (solid lines in bottom panel of Fig. 4). Qualitatively, the main difference shows up in the $\nu_{e}$ spectrum. The initial collective effects induces a "standard" swap only at high energies. Nevertheless, as neutrinos cross the $I$-resonance this split turns into an inverse one, with a swap at low energies. The final result right after the $I$-resonance is analogous to the case of normal mass hierarchy and the $I$-resonance happening first, discussed in Sec. $\mathrm{VA}$ and displayed with dark red solid lines in the bottom panel
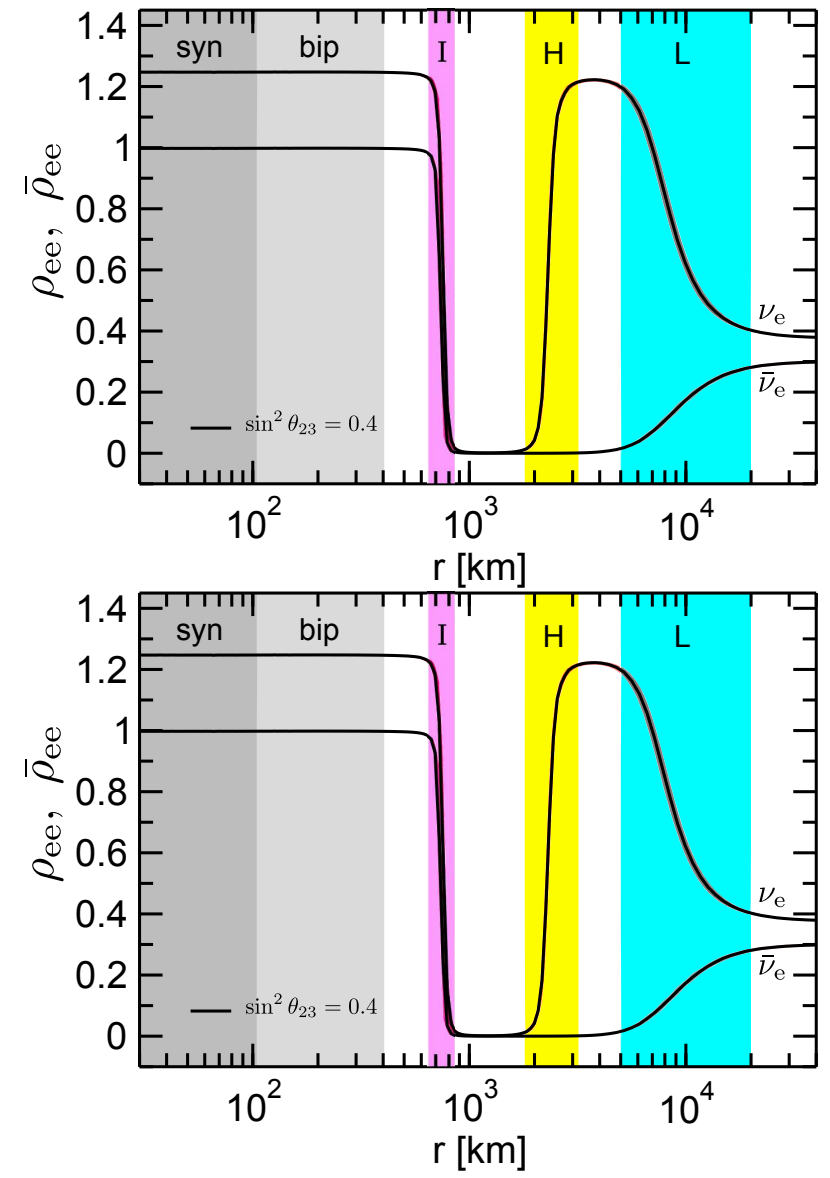

FIG. 10: Same as Fig. 8 but assuming that the collective effects take place before the $I$-resonance.

of Fig. 9 ,

If $\vartheta_{23}$ lies in the second octant then most of $\nu_{e}$ and $\bar{\nu}_{e}$ end up as $\nu_{3}^{\mathrm{m}}$ and $\bar{\nu}_{2}^{\mathrm{m}}$. As can be seen in the solid lines in the bottom panel of Fig. [5 these neutrinos will not traverse the $I$-resonance, except the excess $\epsilon$ of $\nu_{e}$, which stays as $\nu_{1}^{\mathrm{m}}$. These neutrinos will be basically blind to the $I$-, $H$-, and $L$-resonances. The final fluxes will be therefore $\rho_{e e}^{\text {final }}=\sin ^{2} \vartheta_{13}+\epsilon \cos ^{2} \vartheta_{12}$ and $\bar{\rho}_{e e}^{\text {final }}=\sin ^{2} \vartheta_{12}$. This case is represented with dashed lines in the bottom panel of Fig. 10. In the end the final evolution turns out to be similar to that in region IVb.

\section{DISCUSSION}

In the previous sections we have studied the consequences of NSI on the neutrino propagation through the SN envelope taking into account the presence of a neu- 
trino background. We have analyzed the different situations in terms of the non-universal NSI parameter $\varepsilon_{\tau \tau}$ and the density at the neutrinosphere $\lambda_{0}$. Depending on their values we were able to identify four extreme regions of the parameters where the evolution of the neutrinos have a specific pattern.

In a realistic situation, though, we expect to find a combination of these situations depending on the instant considered. As mentioned in Sec. II one expects the value of $\lambda_{0}$ to decrease with time as the explosion goes on. Therefore it is important to look for a time dependence in the neutrino propagation for given values of the NSI parameters. In Fig. 11 we show the composition in terms of mass eigenstates of neutrinos created as $\nu_{e}$ (upper half of the panels) and $\bar{\nu}_{e}$ (lower half of the panels) at the neutrinosphere, when they leave the $\mathrm{SN}$, as function of time for different neutrino mass and mixing schemes and for a given value of $\varepsilon_{\tau \tau}$. The evolution in time shown in each panel is equivalent to consider Fig. 2. fix a value in the $x$ axis corresponding to some $\varepsilon_{\tau \tau}$ and following vertically towards lower values of $\lambda_{0}$. Depending on $\varepsilon_{\tau \tau}$ and the instant considered one can distinguish different regions separated by vertical bands denoting transition phases. The position and the size of these transition bands are not constant but depend on time, as $\mu_{0}$ and $\epsilon$ do. Nevertheless, unless multiangle decoherence is triggered (by e.g. a strong reduction of $\epsilon$ [45]), the sequence of different regimes undergone by the neutrinos is not expected to change drastically.

Let us first discuss the antineutrino case. In the left panel we consider the standard case, i.e. $\varepsilon_{\tau \tau}=0$. On the left we have early times (or large $\lambda_{0}$ ), which corresponds to the region I in Fig. 2 From the previous discussion, we know that for such a case and normal mass hierarchy (upper box) an antineutrino created as $\bar{\nu}_{e}$ leaves the star as $\bar{\nu}_{1}$ whereas in inverted mass hierarchy they escape as $\bar{\nu}_{3}$ due to the adiabatic $H$-resonance for both first and second $\vartheta_{23}$ octants (middle and lower boxes, respectively), see Figs. [3and 4 . At later times, $\lambda_{0}$ becomes smaller and matter can not suppress collective effects any longer, i.e. neutrinos enter region III. These affect only in the inverted mass hierarchy case "canceling" the $H$ resonance conversion and making the initial $\bar{\nu}_{e}$ escape as $\bar{\nu}_{1}$. There is then a time dependence in the survival $\bar{\nu}_{e}$ probability for inverted mass hierarchy but not for the normal one. As can be seen in the panel this behavior does not depend on the $\vartheta_{23}$ octant, see Figs. 3 and 7 , In terms of $\bar{\nu}_{e}$ survival probabilities there is then a transition from $\sin ^{2} \vartheta_{13} \approx 0$ at early times to $\cos ^{2} \vartheta_{12} \approx 0.7$ at later times, the details depending on the specific time evolution of $\lambda(r)$.

Let us now take the middle panel with $\varepsilon_{\tau \tau}=3 \times 10^{-3}$. The situation at early times is the same as in the previous panel, described by the region I. However at intermediate times the situation changes in the case of inverted mass hierarchy. Now the NSI parameters make the evolution go through region IVa before entering eventually region III. The $\mu \tau$-resonance is pushed outside the bipolar region and then the degeneracy between the two $\vartheta_{23}$ octants is broken: for $\vartheta_{23}$ in the first octant $\bar{\nu}_{e}$ leaves as $\bar{\nu}_{1}$ whereas for the second octant they escape as $\bar{\nu}_{2}$, see Figs. 3 and 7 . At later times $\lambda_{0}$ further decreases and the $\mu \tau$-resonance contracts to deeper layers within $r_{\text {syn }}$. Hence neutrinos cross to region III and the $\vartheta_{23}$ octant degeneracy is restored. Concerning the $\bar{\nu}_{e}$ survival probability, as before, there is a transition from $\sin ^{2} \vartheta_{13} \approx 0$ to $\cos ^{2} \vartheta_{12} \approx 0.7$. For $\vartheta_{23}$ in the first octant it is direct, whereas if $\vartheta_{23}$ lies in the second octant it goes from $\sin ^{2} \vartheta_{13} \approx 0$ through $\sin ^{2} \vartheta_{12} \approx 0.3$. As it was discussed in Sec. IVD an analogous effect would arise for a fixed $\vartheta_{23}$ and different signs of $\varepsilon_{\tau \tau}$. For NH there is no time dependence.

Finally, we consider the case where the NSI parameters are large enough, $\varepsilon_{\tau \tau}=5 \times 10^{-2}$, to induce the $I$-resonance, right panel of Fig. 11 Now, at early times neutrino propagation follows the prescription given in region II. For normal mass hierarchy the original $\bar{\nu}_{e}$ leave the star as $\bar{\nu}_{2}$, whereas for inverted they escape as $\bar{\nu}_{1}$ for both octants, see Figs. 5 and 8 . After this phase neutrinos enter the region IVb and collective effects are switched on. For NH they cancel the $I$-induced resonant conversion, whereas for IH they break the degeneracy of the $\vartheta_{23}$ octant for inverted mass hierarchy.

The bottom line is that if $\left|\varepsilon_{\tau \tau}\right| \gtrsim$ a $10^{-3}$ neutrinos cross the region IV during some seconds, and this could help disentangle the $\vartheta_{23}$ octant. If the octant were known one could obtain information about the sign of the nonuniversal NSI parameters.

In the upper half part of the panels we show the same kind of plots but for neutrinos. The main difference with 
respect to antineutrinos is that in the presence of collective effects $\nu_{e}$ are not fully converted like $\bar{\nu}_{e}$. Some fraction of them, corresponding to the excess over $\bar{\nu}_{e}$, remains unaffected. This excess is represented in Fig. 111as a small portion at the right hand side of the corresponding boxes. As discussed, this excess of $\nu_{e}$ translates to a spectral split, so that the flavor spectral swap happens only for some energies. Whether these correspond to the low-energy tail or high-energy tail of the initial spectrum depends on the neutrino oscillation parameters, see bottom panel of Fig. 9. Therefore one could hope to use this additional information to break possible degeneracies between different mass and mixing schemes and different values of the NSI parameters. It is important to note that the results here presented are based on the assumption of a particular choice of the initial neutrino fluxes, with the hierarchy $F_{\nu_{e}}^{R}>F_{\bar{\nu}_{e}}^{R}>F_{\nu_{x}}^{R}$. However, as shown in Ref. [86], a different pattern for the initial fluxes could lead to a more complicated structre of the final spectrum with multilpe spectral splits.

Throughout the analysis we have concentrated on the case of NSI with $d$ quarks. Nevertheless most of the results here presented can be generalized to the case of $u$ quarks and $e$. In the first case the only effect is to shift the position of the $I$-resonance, since the resonance condition is modified to $Y_{e}^{I}=\varepsilon_{\tau \tau} /\left(1-\varepsilon_{\tau \tau}\right)$ [36]. For the case of NSI involving electrons the $I$-resonance is absent. But nevertheless its contribution to increase the value of $Y_{\tau, \mathrm{nsi}}^{\mathrm{eff}}$ would also make the neutrino propagation highly sensitive to the $\vartheta_{23}$ octant and to its own sign, exactly as in the case of $d$ quark.

Last but not least we briefly comment on the possibility to observe the different regimes analyzed. This possibility will be hampered by several uncertainties inherent in SN neutrinos. One is the lack of knowledge on the exact matter profile traversed by the outgoing neutrinos. In our study we assumed a simple power law given in Eq. (5). This density profile will be significantly distorted by the passage of the shock wave responsible for ejecting the whole SN envelope [82, 87, 88]. One of the main effect will be to destroy the adiabaticity of the $H$ resonance, which was assumed in our study. This effect, though, is not always present but depends on the neutrino parameters. Therefore, far from being a problem, the time and energy dependence modulation introduced in the spectra could further help disentangle between the different scenarios here considered [36, 83, 84, 89].

Another important source of uncertainties is our ignorance of the exact initial fluxes $f_{\nu_{\alpha}}^{\mathrm{R}}(E)$. Although the initial fluxes during the first stage of the explosion, the neutronization burst, are rather model independent [90], the expected number of events is very low. Most of the signal is generated later, during the accretion and cooling phases. The spectral features observed in the numerical simulations depend strongly on the properties of the SN. It is therefore necessary to set up strategies combining different observables to be able to pin down the underlying neutrino properties independently of the initial fluxes. For example spectral modulations are expected if neutrinos cross the Earth before being detected [56, 91 94]. One may also take advantage of the time dependence of the matter profiles $\lambda(r)$ and $Y_{e}(r)$ themselves [36].

\section{SUMMARY}

Here we have considered the effect of non-standard neutrino interactions on the propagation of neutrinos through the SN envelope. We adopted a realistic threeneutrino framework, properly taking into account the presence of a neutrino background. We have found that for non-universal NSI parameters exceeding $10^{-3}$, i.e. $\left|\varepsilon_{\tau \tau}\right| \gtrsim 10^{-3}$, the neutrino propagation becomes for some time sensitive to the $\vartheta_{23}$ octant and the sign of $\varepsilon_{\tau \tau}$. In particular, for $\varepsilon_{\tau \tau} \gtrsim 10^{-2}$ an internal $I$-resonance may arise independently of the matter density. For typical values found in simulations this takes place in the same dense-neutrino region above the neutrinosphere where collective effects occur, in particular during the synchronization regime. This resonance may lead to an exchange of the neutrino fluxes entering the bipolar regime. The main implications are (i) bipolar conversion taking place for normal neutrino mass hierarchy and (ii) a transformation of the flux of low-energy $\nu_{e}$, instead of usual spectral swap.

\section{Acknowledgments}

The authors wish to thank S. Pastor and M. A. Tórtola for fruitful discussions. This work is supported by 


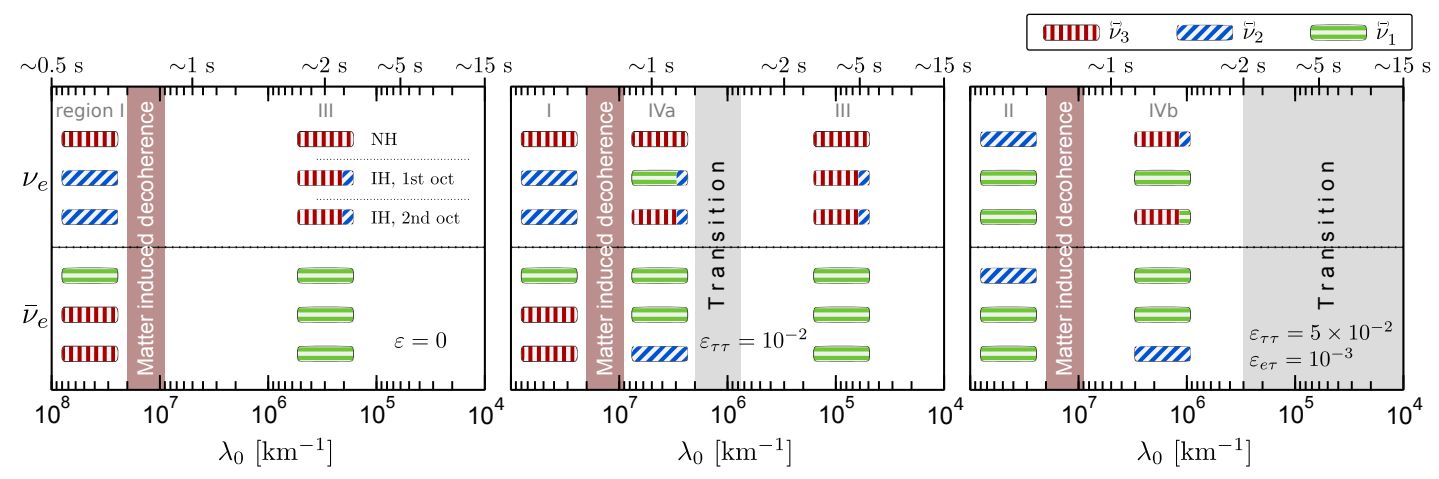

FIG. 11: Relationship between (anti)neutrinos created as $\nu_{e}$ (upper half part) and $\bar{\nu}_{e}$ (lower half part) and the matter eigenstates at the SN surface as function of time for different neutrino mass and mixing schemes and for given values of $\varepsilon_{\tau \tau}=0$ (left), $10^{-2}$ (middle), and $5 \times 10^{-2}$ (right).

the Spanish grants FPA2008-00319/FPA and PROME-

\author{
References
}

TEO/2009/091 and by the DFG (Germany) under grant

SFB-676. A.E. is supported by a FPU grant from the

Spanish Government.

[1] KamLAND collaboration, K. Eguchi et al., Phys. Rev. Lett. 90, 021802 (2003), hep-ex/0212021; T. Araki et al., Phys. Rev. Lett. 94, 081801 (2005).

[2] K2K collaboration, M. H. Ahn, hep-ex/0606032.

[3] MINOS collaboration, P. Adamson et al., 0806.2237.

[4] Super-Kamiokande Collaboration, J. P. Cravens et al. Phys. Rev. D 78, 032002 (2008) arXiv:0803.4312 [hepex]]. S. Fukuda et al., Phys. Lett. B 539, 179 (2002) arXiv:hep-ex/0205075.

[5] SNO collaboration, B. Aharmim et al., Phys. Rev. Lett. 101, 111301 (2008), arXiv:0806.0989; Phys. Rev. C72, 055502 (2005), nucl-ex/0502021; S. N. Ahmed et al., Phys. Rev. Lett. 92, 181301 (2004), nucl-ex/0309004.

[6] GNO collaboration, M. Altmann et al., Phys. Lett. B 490, 16 (2000) arXiv:hep-ex/0006034, SAGE collaboration, J. N. Abdurashitov et al., J. Exp. Theor. Phys. 95, 181 (2002), astro-ph/0204245.

[7] Borexino Collaboration, arXiv:0808.2868

[8] Super-Kamiokande collaboration, Y. Ashie et al., Phys. Rev. D71, 112005 (2005), hep-ex/0501064.

[9] Super-Kamiokande collaboration, Y. Ashie, et al., hep-ex/0404034.

[10] S. Pakvasa and J. W. F. Valle, hep-ph/0301061, Proc. of the Indian National Academy of Sciences on Neutrinos, Vol. 70A, No.1, p.189 - 222 (2004), Eds. D. Indumathi, M.V.N. Murthy and G. Rajasekaran.

[11] T. Schwetz, M. Tortola and J. W. F. Valle, New J. Phys. 10, 113011 (2008), arXiv:0808.2016; for a recent review with references to all groups see, M. Maltoni et al, New J. Phys. 6, 122 (2004) hep-ph/0405172v6] .

[12] J. Schechter and J. W. F. Valle, Phys. Rev. D22, 2227 (1980).

[13] J. W. F. Valle, J. Phys. Conf. Ser. 53, 473 (2006), hep-ph/0608101, Review based on lectures at the Corfu Summer Institute on Elementary Particle Physics in September 2005.

[14] J. Schechter and J. W. F. Valle, Phys. Rev. D24, 1883 (1981), Err. D25, 283 (1982); for recent analyses see O. G. Miranda et al, Nucl. Phys. B 595, 360 (2001) arXiv:hep-ph/0005259; Phys. Rev. D 70, 113002 (2004) arXiv:hep-ph/0406066; Phys. Rev. Lett. 93, 051304 (2004) arXiv:hep-ph/0311014.

[15] L. Wolfenstein, Phys. Rev. D17, 2369 (1978).

[16] Mikheev, S. P. and Smirnov, A. Yu., (Editions Frontières, Gif-sur-Yvette, 1986, p.355.), 86 Massive Neutrinos in Astrophysics and Particle Physics, Proceedings of the Sixth Moriond Workshop, ed. by Fackler, O. and Tran Thanh Van, J.

[17] J. W. F. Valle, Phys. Lett. B199, 432 (1987).

[18] R. N. Mohapatra and J. W. F. Valle, Phys. Rev. D34, 1642 (1986).

[19] J. Bernabeu et al., Phys. Lett. B187, 303 (1987).

[20] G. C. Branco, M. N. Rebelo and J. W. F. Valle, Phys. Lett. B225, 385 (1989); N. Rius and J. W. F. Valle, Phys. Lett. B246, 249 (1990).

[21] F. Deppisch and J. W. F. Valle, Phys. Rev. D72, 036001 
(2005), hep-ph/0406040.

[22] M. Malinsky, J. C. Romao and J. W. F. Valle, Phys. Rev. Lett. 95, 161801 (2005), hep-ph/0506296.

[23] M. Hirsch, S. Morisi and J. W. F. Valle, Phys. Lett. B 679, 454 (2009) arXiv:0905.3056 [hep-ph]].

[24] D. Ibanez, S. Morisi and J. W. F. Valle, Phys. Rev. D 80, 053015 (2009) arXiv:0907.3109 [hep-ph]].

[25] X.-G. He and E. Ma, arXiv:0907.2737.

[26] A. Zee, Phys. Lett. B93, 389 (1980).

[27] K. S. Babu, Phys. Lett. B203, 132 (1988).

[28] For recent work on low-scale generation of neutrino masses and/or non-standard interactions see, for example, D. Aristizabal Sierra, M. Hirsch and S. G. Kovalenko, Phys. Rev. D 77, 055011 (2008) arXiv:0710.5699 [hep-ph]]; T. Ohlsson, T. Schwetz and H. Zhang, arXiv:0909.0455 [hep-ph]; A. Abada, C. Biggio, F. Bonnet, M. B. Gavela and T. Hambye, JHEP 0712, 061 (2007) arXiv:0707.4058 [hep-ph]]; M. Malinsky, T. Ohlsson and H. Zhang, Phys. Rev. D 79, 011301 (2009) arXiv:0811.3346 [hep-ph]]; J. Chakrabortty, A. Dighe, S. Goswami and S. Ray, Nucl. Phys. B 820, 116 (2009) arXiv:0812.2776 [hep-ph]]; Y. Liao, J. Y. Liu and G. Z. Ning, Phys. Rev. D 79, 073003 (2009) arXiv:0902.1434 [hep-ph]]; Z. z. Xing and S. Zhou, arXiv:0906.1757 [hep-ph]; F. Bonnet, D. Hernandez, T. Ota and W. Winter, arXiv:0907.3143 [hep-ph].

[29] F. Bazzocchi et al, arXiv:0907.1262

[30] H. Nunokawa, Y. Z. Qian, A. Rossi and J. W. F. Valle, Phys. Rev. D54, 4356 (1996), hep-ph/9605301.

[31] H. Nunokawa, S. J. Parke and J. W. F. Valle, Prog. Part. Nucl. Phys. 60, 338 (2008), arXiv:0710.0554 [hep-ph]].

[32] ISS Physics Working Group, A. Bandyopadhyay et al., arXiv:0710.4947 [hep-ph].

[33] P. Huber and J. W. F. Valle, Phys. Lett. B523, 151 (2001), hep-ph/0108193.

[34] P. Huber, T. Schwetz and J. W. F. Valle, Phys. Rev. Lett. 88, 101804 (2002), hep-ph/0111224.

[35] H. Nunokawa, A. Rossi and J. W. F. Valle, Nucl. Phys. B482, 481 (1996), hep-ph/9606445.

[36] A. Esteban-Pretel, R. Tomàs and J. W. F. Valle, Phys. Rev. D76, 053001 (2007), arXiv:0704.0032 [hep-ph]].

[37] S. Pastor and G. Raffelt, Phys. Rev. Lett. 89, 191101 (2002), astro-ph/0207281.

[38] R. F. Sawyer, Phys. Rev. D72, 045003 (2005), hep-ph/0503013.

[39] G. M. Fuller and Y.-Z. Qian, Phys. Rev. D73, 023004 (2006), astro-ph/0505240.

[40] H. Duan, G. M. Fuller and Y.-Z. Qian, Phys. Rev. D74, 123004 (2006), astro-ph/0511275.

[41] H. Duan, G. M. Fuller, J. Carlson and Y.-Z. Qian, Phys. Rev. D74, 105014 (2006), astro-ph/0606616.

[42] S. Hannestad, G. G. Raffelt, G. Sigl and Y. Y. Y. Wong, Phys. Rev. D74, 105010 (2006), astro-ph/0608695.
[43] H. Duan, G. M. Fuller, J. Carlson and Y.-Z. Qian, Phys. Rev. D75, 125005 (2007), astro-ph/0703776.

[44] G. G. Raffelt and G. Sigl, Phys. Rev. D75, 083002 (2007), hep-ph/0701182.

[45] A. Esteban-Pretel, S. Pastor, R. Tomàs, G. G. Raffelt and G. Sigl, Phys. Rev. D76, 125018 (2007), arXiv:0706.2498.

[46] G. G. Raffelt and A. Y. Smirnov, Phys. Rev. D76, 081301 (2007), arXiv:0705.1830.

[47] G. G. Raffelt and A. Y. Smirnov, Phys. Rev. D76, 125008 (2007), arXiv:0709.4641.

[48] H. Duan, G. M. Fuller and Y.-Z. Qian, Phys. Rev. D76, 085013 (2007), arXiv:0706.4293.

[49] G. L. Fogli, E. Lisi, A. Marrone and A. Mirizzi, JCAP 0712, 010 (2007), arXiv:0707.1998.

[50] H. Duan, G. M. Fuller, J. Carlson and Y.-Q. Zhong, Phys. Rev. Lett. 99, 241802 (2007), [0707.0290].

[51] H. Duan, G. M. Fuller, J. Carlson and Y.-Z. Qian, Phys. Rev. Lett. 100, 021101 (2008), arXiv:0710.1271.

[52] B. Dasgupta, A. Dighe, A. Mirizzi and G. G. Raffelt, Phys. Rev. D77, 113007 (2008), [0801.1660].

[53] A. Esteban-Pretel, S. Pastor, R. Tomàs, G. G. Raffelt and G. Sigl, Phys. Rev. D77, 065024 (2008), arXiv:0712.1137.

[54] B. Dasgupta and A. Dighe, Phys. Rev. D77, 113002 (2008), arXiv:0712.3798.

[55] H. Duan, G. M. Fuller and Y.-Z. Qian, Phys. Rev. D77, 085016 (2008), arXiv:0801.1363.

[56] B. Dasgupta, A. Dighe and A. Mirizzi, Phys. Rev. Lett. 101, 171801 (2008), arXiv:0802.1481.

[57] R. F. Sawyer, arXiv:0803.4319]

[58] H. Duan, G. M. Fuller and J. Carlson, Comput. Sci. Dis. 1, 015007 (2008), arXiv:0803.3650].

[59] S. Chakraborty, S. Choubey, B. Dasgupta and K. Kar, JCAP 0809, 013 (2008), arXiv:0805.3131.

[60] B. Dasgupta, A. Dighe, A. Mirizzi and G. G. Raffelt, Phys. Rev. D78, 033014 (2008), arXiv:0805.3300.

[61] G. Fogli, E. Lisi, A. Marrone and I. Tamborra, JCAP 0904, 030 (2009), arXiv:0812.3031.

[62] Particle Data Group, C. Amsler et al., Phys. Lett. B667, 1 (2008).

[63] S. P. Mikheev and A. Y. Smirnov, Sov. J. Nucl. Phys. 42, 913 (1985) [Yad. Fiz. 42, 1441 (1985)].

[64] S. P. Mikheev and A. Y. Smirnov, Nuovo Cim. C 9, 17 (1986).

[65] F. J. Botella, C. S. Lim and W. J. Marciano, Phys. Rev. D35, 896 (1987).

[66] E. K. Akhmedov, C. Lunardini and A. Y. Smirnov, Nucl. Phys. B643, 339 (2002), hep-ph/0204091.

[67] A. Mirizzi, S. Pozzorini, G. G. Raffelt and P. D. Serpico, arXiv:0907.3674 [hep-ph].

[68] M. Blennow, A. Mirizzi and P. D. Serpico, Phys. Rev. D78, 113004 (2008), arXiv:0810.2297. 
[69] H. Nunokawa, V. B. Semikoz, A. Y. Smirnov and J. W. F. Valle, Nucl. Phys. B501, 17 (1997), hep-ph/9701420.

[70] J. Gava and C. C. Jean-Louis, arXiv:0907.3947 [hep-ph].

[71] P. S. Amanik, G. M. Fuller and B. Grinstein, Astropart. Phys. 24, 160 (2005), hep-ph/0407130.

[72] P. S. Amanik and G. M. Fuller, Phys. Rev. D 75, 083008 (2007) arXiv:astro-ph/0606607.

[73] N. Fornengo, M. Maltoni, R. Tomàs and J. W. F. Valle, Phys. Rev. D 65, 013010 (2002) arXiv:hep-ph/0108043.

[74] A. Friedland, C. Lunardini and M. Maltoni, Phys. Rev. D 70, 111301 (2004) arXiv:hep-ph/0408264.

[75] A. Friedland and C. Lunardini, Phys. Rev. D 72, 053009 (2005) arXiv:hep-ph/0506143.

[76] F. J. Escrihuela, O. G. Miranda, M. A. Tortola and J. W. F. Valle, Phys. Rev. D 80, 105009 (2009) arXiv:0907.2630 [hep-ph]].

[77] R. F. Sawyer, hep-ph/0408265

[78] T. K. Kuo and J. T. Pantaleone, Rev. Mod. Phys. 61, 937 (1989).

[79] A. S. Dighe and A. Y. Smirnov, Phys. Rev. D62, 033007 (2000), hep-ph/9907423.

[80] G. L. Fogli, E. Lisi, A. Mirizzi and D. Montanino, Phys. Rev. D66, 013009 (2002), hep-ph/0202269.

[81] A. Esteban-Pretel et al., Phys. Rev. D78, 085012 (2008), arXiv:0807.0659.
[82] R. C. Schirato, G. M. Fuller, astro-ph/0205390

[83] G. L. Fogli, E. Lisi, D. Montanino and A. Mirizzi, Phys. Rev. D68, 033005 (2003), hep-ph/0304056.

[84] R. Tomas et al., JCAP 0409, 015 (2004), astro-ph/0407132.

[85] M. T. Keil, G. G. Raffelt and H.-T. Janka, astro-ph/0208035

[86] B. Dasgupta, A. Dighe, G. G. Raffelt and A. Y. Smirnov, Phys. Rev. Lett. 103, 051105 (2009), [0904.3542].

[87] G. L. Fogli, E. Lisi, A. Mirizzi and D. Montanino, JCAP 0606, 012 (2006), hep-ph/0603033.

[88] A. Friedland and A. Gruzinov, astro-ph/0607244.

[89] J. Gava, J. Kneller, C. Volpe and G. C. McLaughlin, Phys. Rev. Lett. 103, 071101 (2009), arXiv:0902.0317.

[90] M. Kachelriess et al., Phys. Rev. D71, 063003 (2005), astro-ph/0412082.

[91] C. Lunardini and A. Y. Smirnov, Nucl. Phys. B616, 307 (2001), hep-ph/0106149.

[92] A. S. Dighe, M. T. Keil and G. G. Raffelt, JCAP 0306, 005 (2003), hep-ph/0303210.

[93] A. S. Dighe, M. T. Keil and G. G. Raffelt, JCAP 0306, 006 (2003), hep-ph/0304150.

[94] A. S. Dighe, M. Kachelriess, G. G. Raffelt and R. Tomas, JCAP 0401, 004 (2004), hep-ph/0311172. 